Catalogue of the Doane Collection of Shells w...u...

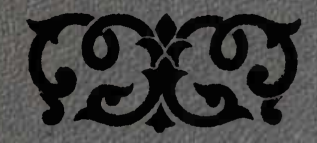

Presented by Mrs. Rose Delano to the Marion Natural History Society.

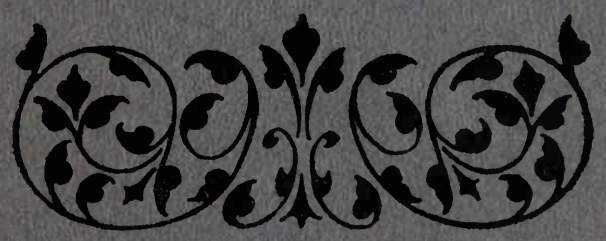

THE WAMEHAM PAKES 


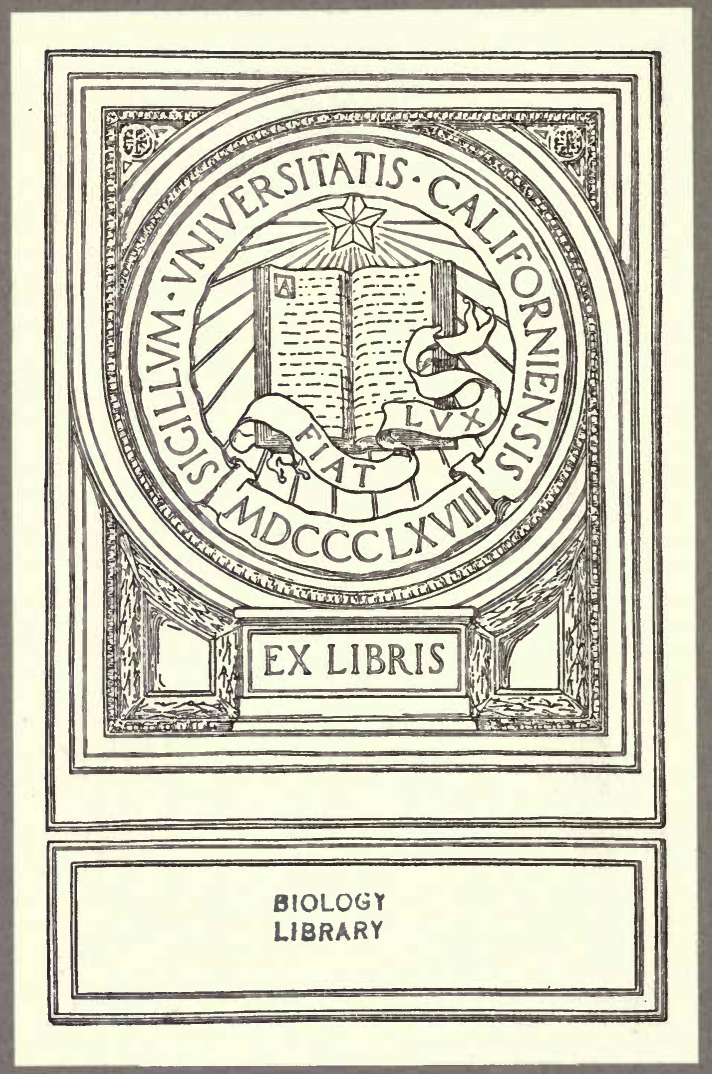





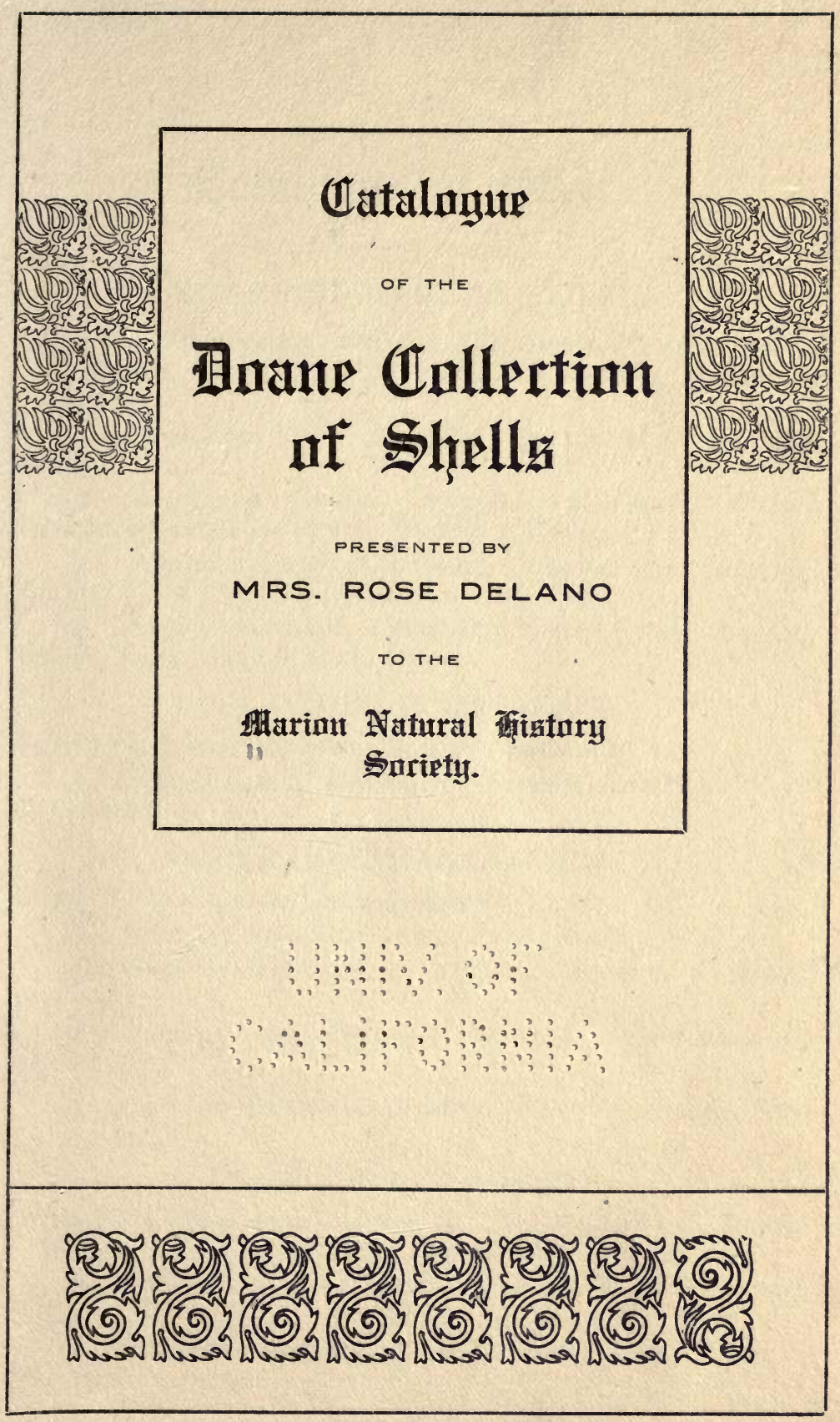


QL406

M35

BIOLOGY

LIBRARY 


\section{Uatalnute of Shrella.}

\section{SUB-KINGDOM II. MOLLUSCA.}

Class I. Cephalopoda. Order I. Dibranchiata. Sub-order I. Octopoda. Family 3. Argonautidae. Genus Argonauta (Linn.). China.

I. Argonauta hians (Solander), South Atlantic Ocean,

2. Argonauta Nouryi (Lorois), Equatorial Pacific from Marquesas Isles to near Peruvian Coast.

3. Argonauta Argo (Linnaeus), Tropical Pac., Ind., and At. O.; G of Cal.; Med. Sea; C. of G. Hope.

4. Argonauta nodosa (Solander) Brazil, New Zealand, Indian Ocean, Cape of Good Hope.

\section{SUB-ORDER II DECAPODA.}

Family 14 Spirulidae, Genus Spirula (Lamarck).

5. Spirula Peronii (Lamarck), Tropical Atlantic and Pacific Oceans.

ORDER II TETRABRANCHIATA.

Family I. Nautilidae, Genus Nautilus (Breyn).

6. Nautilus Pompilius (Linn), Polynesia.

7. Nautilus stenomphalus, (Sowb.), Eastern Archipelago. etc.

8. Nautilus macromphalus (Sowb.) New Caledonia,

9. Nautilus umbilicatus (Lister.), Solomon Islands, New Ireland, etc.

\section{CLASS II GASTEROPODA.}

Order I Prosobranchiata, Section A, Siphonostomata, Family Muricidae, Sub-family Muricinae, Genus Murex (Linn.) China.

10. Murex scolopax (Dillw), Red Sea, Indian Ocean,

I1. Murex tenuispina (Lam.), Indian Ocean, Japan, Northern Australia. 

China.

I2. Murex ternispina (Lam.), Indian Ocean, Japan,

13. Murex brevispina (Lam.), Red Sea, Northern Australia, South Africa.

14. Murex recurvirostris (Brod.), West Indies, West Coast of Central Americal. China.

I5. Murex haustellum (Linn.), Red Sea, Philippines,

SUB-GENUS PTERONOTUS (SWAINSON).

r6. Murex triqueter (Born), Red Sea, Paumotus Is.

I7. Murex triformis (Reeve), Port Lincoln, Australia. Wales.

I8. Murex acanthopterus (Lam.), Watson's Bay, N. S.

19. Murex hemitripterus (Lam.), Senegambia.

20. Murex pinnatus (Wood), Chinese Seas.

SUB-GENUS CHICOREUS (MONTF.)

2r. Murex palma-rosae (Lam.), Indian Ocean.

22. Murex torrefactus, (Sowb.), China. Japan.

23. Murex adustus (Lam.); Indian Ocean, Philippines,

24. Murex Penchinata (?) (Croese), Liu Tschiu Is.

25. Murex anguliferus (Lam.), Red Sea, Indian Ocean, Isle of Bourbon.

ro7. Murex Senegalensis (Gmel.), Senegal, Rio Janeiro.

26. Murex ramosus (Linn.), Red Sea, Indian Ocean, Hong Kong, Australia, Central Pacific Ocean.

27. Murex elongatus (Lam.), Indian Ocean, China.

28. Murex brevifrons (Lam.), Red Sea, Indian Ocean, China, Brazil, West Indies.

29. Murex pomum (Gmel.), West Indies.

SUB-GENUS RHINOCANTHA (H. \& A. AD.) of Africa.

30. Murex brandaris (Linn.), Mediterranean, West Coast

3r. Murex cornutus (Linn.), West Coast of Africa.

SUB-GENUS PHYLLONOTUS (SWAINSON.)

32. Murex rosarium (Chemn.), Senegal, Fernando Po.

33. Murex regius (Wood), Panama to Mazatlan.

34. Murex bicolor (Val.), Panama to Guaymas. 

Africa.

35. Murex saxatilis (Lam.), Indian Ocean, West Coast of

36. Murex Stainforthii (Reeve), North Australia.

37. Murex faciatus (Sowb.), West Coast of Africa.

150. Murex nitidus (Brod.) Mazatlan.

38. Murex radix (Gmel.), Panama.

39. Murex trunculus (Linn.), Mediterranean Sea.

40. Murex spinicostata (Val.), West Indies.

4I. Murex hexagonus (Lam.), West Indies.

45. Murex pauxillus (A. Ad.), Mazatlan.

SUB-GENUS CEROSTOMA (CONRAD) Cal., Asia?

42. Murex foliatus (Martyn), Sitka to Santa Barbara,

SUB-GENUS OCINEBRA (LEACH)

43. Murex erinaceus (Linn.), Norway to Black Sea, Azores, Madeira.

SUB-GENUS VITULARIA (SWAINS)

43 I-2. Murex miliaris (Gmel.), West Coast of Africa. SUB-GENUS VITULARIA (SWAINSON)

44. Murex salebrosa (King), Mazatlan to Panama. GENUS TROPHON (MONTF.)

46. Trophon Clathratus (Linn.), Spitzbergen, Great Britain, Iceland, Mass.. Japan, North West C. of America. Waters.

47. Trophon clavatus (Sars.), Northern European

GENUS EUPLEURA (H. \& A. AD.)

49. Eupleura candata (Say), Massachusetts to Florida

50. Murex Angasi (Tryon), South Australia.

SUB-FAMILY PURPURINAE.

Genus Purpura (Brugiere)

75. Purpura patula (Linn.), Gulf of Cal., Panama, Philippines, West Indies.

51. Purpura persica (Linn.), Philippines. SUB-GENUS PURPURELLO (DALL.) Isles.

52. Purpura columnellaris (Lam.), Mazatlan, Gallapagos 
SUB-GENUS TRIBULUS (KLEIN) California.

53. Purpura planospira (Lam.), Gallapagos Isles, Lower

56. Purpura aperta (Blainv.), W. C. of Central America, Sandwich Isles.

SUB-GENUS THALESSA (H. \& A. AD.)

76. Purpura hippocastaneum (Lam.), Zanzibar, Indian Ocean, Philippines, Cent. Pac., Paumotus.

77. Purpura tumulosa (Reeve), Zanzibar? Japan.

78. Purpura pica (Blainv.), China Sea, Philippines, Central Pacific Ocean. etc.

79. Purpura armigera (Chemn.), Philippines, Panmotus,

8o. Purpura deltoidea (Lam.), West Indies, Florida.

54. Purpura melones (Duclos), Pacific Coast of Central America.

55. Purpura mancinella (Linn.), Japan, Singepore, Australia, Fiji Is., W. C. of Central America, Peru. cension Is.

57. Purpura neritoidea (Linn.), Cape Verde Is., As-

58. Purpura bufo (Lam.), Philippines. SUB-GENUS STRAMONITA (SCHUM)

59. Purpura Consul (Lam.), Philippines.

6o. Purpura haemastoma (Linn.), Mediterranean Sea, W. C. of Africa, W. I., W. C. of Mexico.

6I. Purpura haemastoma. Form, Undata (Lam.), West Indies, W. C. of America, East Indies.

63. Purpura haemastoma. Form, Bicostalis (Lam.), East Indies, West Columbia.

\section{SUB-GENUS TROCHIA (SWAINS)}

8I. Purpura cingulata (Linn.), Cape of Good Hope.

62. Purpura succincta (Martyn), Australia, New Zealand, C. of G. H.

\section{SUB-GENUS POLYTROPA (SWAINSON)} Boreal Seas,

48. Purpura lapillus. Form, crispata, (Linn.)(Chemn.),

GENUS JOPAS (H. \& A. AD.

64. Jopas sertum (Brug.). Red Sea to Paumotus Is. 
GENUS VEXILLA (SWAINSON)

183. Vexilla taeniata (Powis), Philippines to Paumotus, Sand wich Is.

I84. Vexilla vexillum (Chem.), Philippines, Sandwich Is.

GENUS RICINULA (LAM.) motus.

65. Ricinula hystrix (Linn.), Sandwich Is., Fiji Is., Pau-

66. Ricinula hystrix. Var, clathrata (Lam.), Central Pacific.

67. Ricinula iodostoma (Lesson), Tahiti, Sts. of Macassar, New Zealand.

68. Ricinula horrida (Lam.), Philippines to Sandwich Is.

69. Kicinula ricinus (Linn.), Red Sea, Benguela, Natal, Guinea, Japan, Philippines, New Zealand, Polynessa.

7o. Ricinula digitata (Lam.), Central Pacific Isles.

71. Ricinula digitata, Var., lobata (Blainv.), Central Pacific Isles.

SUB-GENUS SISTRUM (MONTF.)

72. Ricinula morus (Lam.), Polynesia.

73. Ricinula morus, Var., stratus (Pease), Paumotus.

74. Ricinula tuberculatus (Blainv.), Japan, Philippines to Sandwich Isles. motus.

185. Ricinula Anaxares (Duclos), Natal, Australia, Pau-

\section{GEMS MONOCEROS (LAM.)}

82. Monoceros municatum (Brod.), Panama to Mazatlan.

83. Monoceros lugubre (Sowb.) Pacific and Gulf Coasts of Lower California.

108. Monoceros calcar (Martyn), Cape Horn to Chili.

GENUS CONCHOLEPAS (LAM.)

84. Concholepas Peruvianus (Lam.), W. C. of South America from Peru to Patagonia.

\section{GENUS CUMA (HUMPH.)}

85. Cuma carinisera (Lam.), Philippines, Ascension Is., St. Helena.

86. Cuma coronata (Lam.), West Coast of Africa.

GENUS RAPANA (SCHUM.)

I09. Rapana bulbosa (Sol.) China, Japan, Philippines. 
GENUS RHIZOCHILUS (STEENSTRUP)

Sub-Genus Coralliophila (H. \& A. Ad.)

87. Coralliophila neritoidea, Central Pacific.

136. Coralliophila galea (Chemn.), Philippines, Central Pacific, West Indies.

SUB-GENUS GALEROPSIS (HUPE)

88. Rhizochilus madreporarum (Sowb.), Indian Ocean, Japan, Central Pacific.

\section{FAMILY TRITONIDAE}

Genus Triton (Montfort)

89. Triton Tritonis (Linn.), Philippines. New Zealand, Japan, Indian Ocean, Polynesian Is.

\section{SUB-GENUS SIMPULUM (KLEIN)}

9o. Triton olearium (Linn.), Mediterranean Sea, At. C. of Europe and Africa, W. I. to Brazil, Poly., Austr., Japan.

91. Triton pilearis (Linn.), Red Sea, Natal, Japan, Austr., Phil., Sandw. Is., Flor., W. I., Brazil.

II3. Triton rubecula (Linn.), Red Sea, Phil., Cent. Pac., Sandw. Is., St. Thomas, W. I.

92. Triton chlorostomus (Lam.), Red Sea, Phil., Cent. Pac., Sandw. Is., West Indies.

93. Triton clandestinus (Lam.), Australia.

94. Triton Spengleri (Lam.), S. Australia, Chatham Is.

SUB-GENUS CYMATIUM (BOLTEN) ADAMS

95. Triton femorale (Linn.). West India Islands.

96. Triton lotorium (Linn.), Red Sea, Ind. O., Phil., Cent. Polynesia.

\section{SUB-GENUS GUTTURNIUM (ADAMS)}

I 12. Triton cynocephalus (Lam.), W. I., Philippines.

97. Triton trilineatus (Reeve), Red Sea, Phil., China.

98. Triton Sinensis (Reeve), Chinese Sea.

99. Triton candatus (Gmel.), Chinese Sea.

I0o. Triton tripus (Lam.), Indian Ocean, China.

I I I. Triton vespaceus (Lam.), Austr., Viti Is., Sandw. Is., W. I.

IOI. Triton Pfeifferianus (Reeve), Philippines.

I I0. Triton encausticus (Reeve) I. Ticao, Philippines. 
SUB-GENUS EPIDROMUS (KLEIN) ADAMS

102. Triton maculosus (Gmel.), Mauritius, Phil., Amboina, Red Sea.

103. Triton distortus (Schubert and Wagner), Indo-Pac. Ocean, Polynesia.

104. Triton obscurus (Reeve), Indian Ocean, Paumotus, W. I., Cape Verde Is.

\section{SUB-GENUS PRIENE (HAND A. AD.)}

186. Triton scaber (King.), Valparaiso, Bolivia, Arctic America, California.

\section{GENUS DISTORSIO (BOLTEN)}

105. Distorsio anus (Linn.), Red Sea, Indian Ocean, Phil., Society Is.

106. Distorsio cancellinus (Roissy), Ceylon, China, Phil., W. I., Monte Christi, W. Columbia.

GENUS RANELLA (LAMARCK)

I14. Ranella spinosa (Gam.), Red Sea, Ind. O., Phil., Mauritius.

I15. Ranella foliata (Brod.), Mauritius.

i 6. Ranella crumena (Lam.), Ceylon, Philippines.

SUB-GENUS LAMPAS (SCHUM.)

I 7. Ranella lampas (Linn.), Red Sea, Ind. O., Phil., Viti Is., Mauritius.

I 18. Ranella bufonia (Gmel.), Red Sea, Phil., Seychelles, I. Bourbon, Paumotus, etc.

I I9. Ranella bufonia Var., venustula (Reeve), same localities.

I87. Kanella ventricosa (Brod.), Callao, Peru.

1 20. Ranella pustulosa (Reeve), Ascension I., At. O., $280 \mathrm{~m}$. N. W. of St. Helena.

I21. Ranella granifera (Lam.), Red Sea, Natal, Paumotus, Phil., N. E. coast of Australia.

SUB-GENUS ARGOBUCCINUM (KLEIN.)

122. Ranella gigantea (Lam.), Mediterranean Sea.

123. Ranella pulchra (Gray), Philippines, Japan.

124. Ranella argus (Gmel.), C. of G. H., Chili, New Zealand, Indian Ocean. 


\section{FAMILY FUSIDAE.}

Sub-Family Fusinae, Genus Fusus (Lam.)

125. Fusus proboscidiferus (Lam.), Australia.

I26. Fusus colus (Linn.), Ceylon, Tonga Tabou.

127. Fusus nicobaricus (Lam.), Japan, Philippines.

128. Fusus tuberculatus (Lam.), Red Sea, Indian Ocean.

r29. Fusus longissimus (Gmel.), Ceylon.

I3o. Fusus undatus (Gmel.), Central Polynesia.

GENUS CLAVELLA (SWAINSON).

I31. Clavella serotina (Hinds), Nukahiva, Marquesas. SUB-FAMILY FASCIOLARIINAE.

Genus Fasciolaria (Lam.)

132. Fasciolaria tulipa (Linn.), West Indies, South coast of U. S. from N. Carolina.

I33. Fasciolaria filamentosa (Lam.), Red Sea, Ceylon, Phil., Austr., Cen. Polynesia.

I34. Fasciolaria aurantiaca? (Lam.), Coast of Brazil ? Cape of Good Hope?

135. Fasciolaria trapezium (Linn.), Phil., Java, China, Red Sea, Auckland Is. ?

\section{GENUS PERISTERNIA (MORCH.)}

138. Peristernia ustulata (Reeve), New Caledonia, Viti Is. Mauritius, Indian Ocean.

\section{GENUS LATIRUS (MONTF.)}

I39. Latirus polygonus (Gmel.), Phil., Mascarene Is., Red Sea, Cen. Pac., I. of Ticao. Is.

I 5 I. Latirus amplustris (Mart.), Is. of Annaa, Ascension

I40. Latirus ceratus (Gray), Galapagos Is., Panama, Mazatlan.

I4I. Latirus infundibulum (Gmel.), West Indies.

142. Latirus filosus (Schubert and Wagner), Prince's Is., Senegal.

143. Latirus nodatus (Martyn), Sandwich and Viti Is.

I44. Latirus craticulatus (Linn.), Red Sea, Ind. O., Phil., Cent. Poly., I. of Bourbon.

145. Latirus turritus (Gmel.), Red Sea, Phil., Austr., Cent. Poly., I. of Bourbon. 
146. Latirus prismaticus (Martyn), Central Polynesia.

GENUS LEUCOZONIA (GRAY)

I37. Leucozonia cingulifera, (Lam.), West Africa, West Indies, Florida, Brazil.

147. Leucozonia ocellata (Gmel.), West Indies.

148. Leucozonia cingulata (Lam.), Panama to Mazatlan. south extremity of Florida.

SUB-GENUS LAGENA (SCHUM). Is.

149. Leucozonia smaragdula (Linn.), Philippines, Viti

FAMILY BUCCINIDAE.

Sub-Family Melongeninae, Genus Melongena (Schum.) Mazatlan.

152. Melongena patula (Brod. and Sowb.), Panama to

153. Melongena melongena (Linn.), West Indies. Indies.

154. Melongena corona (Gmelin.), Florida and West

155. Melongena galeodes (Lam.) Red Sea, Ind. O., Singapore, China, Phil.

156. Melongena pallida (Brod. and Sowb.), Mazatlan, W. coast of Cen. America?

157. Melongena fusiformis (Blainv.), St. Elena, W. Columbia, Peru.

158. Melongena pugilina, (Born.), Indian Ocean.

159. Melongena paradisiaca (Reeve), Red Sea, Ceylon, Mozambique, Natal.

I6o. Melongena cochlidium (Linn.), Indian Ocean, Raines Is., Forres Sts.

I6I. Melongena morio (Linn.), W. coast of Africa, West Indies, Brazil.

162. Melongena squamosa (Brod.), Payta, Peru.

SUB-FAMILY NEPTUNIINAE.

Genus Neptunea (Bolten).

163. Neptunea antiqua (Linn.), Cornwall, Shetland, North Sea, At. coast of France.

164. Neptunea decemcostata (Say.), Mass. Bay, Maine, Nova Scotia, etc.

GENUS FULGUR (MONTFORD)

165. Fulgur carica (Gmel.), Cape Cod to Florida. 
166. Fulgur carica Var :- eliceans (Montf.), Georgia and Florida.

167. Fulgur preversus (Linn.), Florida.

SUB-GENUS SYCOTYPUS (BROWN) [GILL]

168. Fulgur canaliculatus (Linn.), Cape Cod to Florida.

169. Fulgur Pryum (Dillw.), Florida.

GENUS TUDICLA BOL TEN).

I 7o. Tudicla spirillus (Linn.), Indian Ocean.

SUB-FAMILY PISANIINAE

Genus Pisania (Bivona)

I7I. Pisania pusio (Linn.), West Indies, Central America. GENUS EUTHRIA (GRAY.)

I72. Euthria cornea (Linn.), Mediterranean. GENUS CANTHARUS (BOLTEN)

173. Cantharus melanostoma (Sowb.), Ceylon.

I 74. Cantharus erythrostoma (Reeve), Ceylon, Japan.

175. Cantharus undosus (Linn.), Malacca, Australia, Phil., Viti and Paumotus Is.

r89. Cantharus distortus (Gray), W. Columbia, Panama.

SUB-FAMILY BUCCININAE

Genus Buccinum (Linn.)

176. Buccinum undatum (Linn.), Arctic O., North C. to France, Newfoundland to New Jersey.

GENUS COMINELLA (GRAY)

177. Cominella maculata (Martyn), New Zealand.

SUB-FAMILY EBURNINAE

Genus Eburna (Lam.)

178. Eburna Zeylanica (Brug.), Ceylon.

179. Eburna lutosa (Lam.), China.

I80. Eburna areolata (Lam.), Ceylon, China Sea.

ISI. Eburna spirata (Lam.), Ceylon, Philippines. SUB-GENUS ZEMIRA (H. \& A. AD.)

190. Eburna Australis (Sowb.), Port Jackson, Australis. SUB-FAMILY PHOTINAE

Genus Phos. (Mont.) 
182. Phos. senticosus (Linn.), Phil., New Caledonia, Amboina, Andaman Is.

GENUS NASSARIA (LINK.)[H. \& A. AD.]

188. Nassaria acuminata (Reeve), China S., Ind. O. FAMILY NASSIDAE.

Genus Northia (Gray)

191. Northia serrata (Dufresne), Panama, St. Elena, W. Columbia.

GENUS BULLIA (GRAY)

192. Bullia laevissima (Gmel.), Cape of Good Hope. SUB-GENUS BUCCINANOPS (D'ORB.) gonia.

193. Bullia cochlidium (Kiener.), Peru and Brazil, Pata-

194. Bullia armata (Gray), Rio Negro, Patagonia.

SUB-GENUS PSEUDOSTROMBUS (KLEIN.)

195. Bullia turrita (Gray) (?)

196. Bullia vittata (Linn.), Ceylon, Zanzibar.

I97 Bullia Belangeri (Kiener), Aracan, Ceylon.

GENUS NASSA (LAM.) pines, etc.

198. Nassa coronata (Brug.), Madagascar, Java, Philip-

199. Nassa arcularia (Linn.), Philippines, Viti Is. SUB-GENUS ARCULARIA (LINK.)

200. Nassa granifera (Kiener), Philippines to Cen. Polynesia, Isle of Bourbon.

SUB-GENUS ALECTRION (MONTF.)

201. Nassa glans (Linn.), Japan, Philippines, Australia.

202. Nassa glans Var:- Elegans (Kiener), So. Australia.

203. Nassa glans Var:- suturalis (Lam.), Philippines, Australia, New Caledonia.

204. Nassa hirta (Kiener), Phil., Austr., Poly., Ind. O.

205. Nassa scalaris (A. Ad.), I. of Corrigidor, Phil.

206 Nassa papillosa (Linn.), Phil. Is., Cent., Poly.

SUB-GENUS ZEUXIS (H. \& A. Ad.)

Poly.

207. Nassa taenia (Gmel.), Ceylon, Singapore, Austr., 
208. Nassa serotina (A. Ad.), Australia, C. of G. H. SUB-GENUS PRONTIS (H. \& A. Ad.)

209. Nassa vibex (Say.), W. I., Chesapeake Bay to Rio Janeiro, Brazil.

SUB-GENUS HINNA (LEACH).

2 ro. Nassa concinna (Powis), Poly., Austr. SUB-GENUS NIOTHA (H. \& A. AD.)

21 1. Nassa gemmulata (Lam.), Phil, Sts. of Sunda. SUB-GENUS TRITIA (RISSO)

21 2. Nassa trivittata (Say.), Mass., Florida. SUB-GENUS ILYANASSA (STIMPSON)

213. Nassa obsoleta (Say.), Mass., Florida. GENUS NERITULA (PLANCUS)

2I3 I-2 Neritula neritea (L.), Mediterranean.

2 3 3-4 Neritula pellucida (Risso), Mediterranean. FAMILY TURBINELLIDAE

Genus Turbinella (Lam.)

214. Turbinella pyrum (Linn.), Ceylon.

215. Tubinella ovoidea (Keiner), Bahia, Coast of Brazil.

216. Turbinella scolymus (Gmelin), Bahia, Brazil, West Indies?

\section{GENUS VASUM (BOLTEN)}

217. Vasum muriaticum (Born), West Indies, Panama, Mazatlan.

218. Vasum rhinoceros(Gmelin), Zanzibar, Carthagena, N. G., Bahia, Brazil.

219. Vasum ceramicum (Linn.), Phil., Poly.

220. Vasum tubinellum (Linn.), Red Sea, Moluccas, Phil, Mauritius, Cen. Poly.

\section{FAMILY VOLUTIDAE}

Genus Cymbium (Klein) Africa.

22 I Cymbium proboscidale (Lam.), West Coast of

222. Cymbium cisium (Lam.), West Africa.

223. Cymbium Neptuni (Gmel.), West Africa. 
GENUS MELO (HUMPHREY).

224. Melo Indica (Gmel.), Indian Ocean.

225. Melo Aethiopica (Linn.). Ind O., Manilla, Japan.

226. Melo dialdema (Lam.), Ind. O., Austr.

GENUS VOLUTA (LINN.)

Sec. I. Voluta (Gray.)

227. Voluta musica (Linn.), West Indies.

228. Voluta virescens (Solander), W, coast of Africa.

229. Voluta Hebraea (Linn.), W. coast of Africa, Brazil. SECTION II, HARPULA (SWAIN)

230. Voluta interpuncta (Martyn), Ceylon, Indian O.

231. Voluta vexillum (Lam.), Indian $O$.

SECTION III, FUGORARIA (SCHURMACHER).

232. Voluta rupestris (Gmel.), China, Japan.

SECTION IV, VESPERTILIO (LINN.)

233. Voluta vespertilio (Linn.), Philippines, Moluccas.

234. Voluta pulchra (Sowerby), N. and N. E. Austr.

235. Voluta nivosa (Lamark), W. Coast of Austr.

236. Voluta norrisii (Gray), W. coast of Austr. Guinea.

237. Voluta rutila (Brod.), N. E. coast of Austr., New

SECTION V, ARELICA (H. \& A. AD.)

238. Voluta Deshayesii (Reeve.), New Caledonia.

239. Voluta imperialis (Lam.), Phil. Is.

240. Voluta scapha (Gmel.), Singapore.

Florida.

241. Voluta Junonia (Chemn.), E. and W. coasts of

242. Voluta Grayae (Crosse), N. W. coast of Austr SECTION VI, AMORIA (Gray). Tasmania.

243. Voluta undulata (Lam.), E., W. and S. Austr.,

244. Voluta Turneri (Gray), N. E. Austr., W. Austr.

245. Voluta volva (Gmel.), W. Austr.

246. Voluta zebra (Leach.), E. coast of Austr.

247. Voluta reticulata (Reeve), W. Austr.

248. Voluta praetexta (Reeve), N. Austr. 
SECTION VII, ALCITHOA (H. \& A. AD.)

249. Voluta Pacifica (Solander), New Zealand.

250. Voluta fusiformis (Swainson), Tasmania, So. Austr.

SECTION IX, VOLUTELLA (D'ORBIGNY).

251. Voluta angulata (Swainson), Mouth of the Rio de la Plata to Patagonia.

\section{GENUS LYRIA (GRAY)}

26o. Lyria deliciosa (Nontv.), New Caledonia.

261. Lyria Delessertiana (Petit.), Madagascar.

FAMILY MITRIDAE.

Genus Mitra (Lam).

252. Mitra episcopalis (Linn.), Ceylon, Philippines, Polynesia.

253. Mitra papalis (Linn.), Kingsmill, Caroline and P:aumotus Isles.

254. Mitra pontificalis (Lam.), Polynesia.

255. Mitra cardinalis (Gmel.), Ceylon, Polynesia.

361. Mitra versicolor (Martyn), Red Sea, Zanzibar, Madagascar, Poly.

256. Mitra variegata (Recve), Red Sea, Mauritius, Phil.

257. Mitra Fergusoni (Sowb.), Panama.

258. Mitra Florida (Gould), Florida, Phil. Is.

363. Mitra Barbadensis (Gmelin), West Indies.

362. Mitra contracta (Swainson), Red Sea, Phil, Poly. Africa.

291. Mitra cornicula (Linn.), Med. S., At. C. of N.

259. Mitra ebenus (Lam.), Med. S., N. At. C. of Africa. SECTION SWAINSONIA (H. \& A. AD.).

262. Mitra casta (Lam.), Zanzibar, Mauritius, Polynesia.

263. Mitra fissurata (Lam.), Red S., Mauritius, Zanzibar. SECTION SCABRICOLA (SWAINSON).

264. Mitra vitellina (Gould), East Indies.

265. Mitra crenifera (Lam.), Mauritius, Manilla, Red S., Ind. $\mathrm{O}$

266. Mitra scabriuscula (Linn.), Phil., Viti Is. SECTION CANCILLA (SWAINSON)

267. Mitra filaris (Linn.), Mauritius, Philippines, Poly- 
nesia.

268. Mitra carnicolor (Reeve), Philippines.

269. Mitra cucumenina (Lam.), Polynesia. SECTION CHRYSAME (H. \& A. AD.)

270. Mitra ambigua (Swainson) Var :- fulva (Swains.), Polynesia.

271. Mitra adusta (Lam.), Polynesia.

272. Mitra coronata (Lam.), Red Sea, Phil., Sandw. Is., Mauritius.

273. Mitra ferruginea (Lam.), Phil., Poly., Mauritius.

SECTION STRIGATELLA (SWAINSON)

274. Mitra astricta (Reeve), Sandw. Is.

275. Mitra auriculoides (Reeve), Polynesia.

276. Mitra limbifera (Lam.), Philippines, Polynesia.

277. Mitra scutulata (Lam.), Philippines, Polynesia.

278. Mitra litterata (Lam.), Red Sea, Java, Mauritius, S. Africa, Phil., Poly.

279. Mitra paupercula (Linn.), Red Sea, E. Africa, Ind. O., Phil., Poly.

280. Mitra virgata (Reeve), Polynesia.

281. Mitra retusa (Lam.), Polynesia.

GENUS MITROIDEA (PEASE).

364. Mitroidea infecta (Reeve), Mauritius, Paumotus.

GENUS TURRICULA (KLEIN).

282. Turricula regina (Sowb.), Moluccas.

283. Turricula taeniata (Lam.], Phil., Moluccas, Poly.

284. Turricula sanguinea (L.) Var :-stigmataria (Lam.)

Philippines, Mauritius, Polynesia.

365. Turricula vulpecula (Linn.), Moluccas, Phil., Poly.

286. Turricula caffra (Linn.), Isle of Ticao, Phil.

287. Turricula plicata (Lam.), Philippines.

288. Turricula corrugata (Lam.), Ind. O., Phil., New Guinea, Australia.

285. Turricula obeliscus (Reeve), Philippines, Andaman Is., Viti Is.

SECTION COSTELLARIA (SWAINSON).

289. Turricula exasperata (Gmelin), Red S., Ind. O., Java, Phil., Poly. 


\section{SECIION PUSIA (SWAINSON).}

290. Turricula variata (Reeve), Paumotus, and Viti Is.

GENUS CYLINDRA (SCHUM).

292. Cylindra dactylus (Linn.), Philippines, Polynesia.

GENUS IMBRICARIA (SCHUMACHER). nesia.

293. Imbricaria conica (Schumacher), Philippines, Polynesia.

294. Imbricaria Vanikorensis (Quoy), Philippines, Poly-

366. Cylindra nucea (Gronov.), Polynesia.

367. Cylindra crenulata (Gmelin), Red Sea, Ind. O., Mauritius, Phil., Poly.

FAMILY MARGINELLIDAE.

Genus Marginella (Lamark).

295. Marginella glabella (Linn.), West Coast of Africa, Canary Is.

\section{SECTION GLABELLA (SWAINSON).}

296. Marginella Adansoni (Kiener), Senegal and Gambia, N. Africa.

297. Marginella limbata (Lam.), N. Africa. mania.

298. Marginella formicula (Lam.), S. Australia, Tas-

\section{SECTION PRUNUM (MARTINI) [ADAMS].}

299. Marginella marginata (Born), Senegal, W. Africa, W. I., Brazil.

300. Marginella curta (Sowb.), W. C. of S. America.

3oi. Marginella prunum (Gmel.), W. I, Panama, Brazil, Sierra Leone.

\section{SECTION CRYPTOSPIRA (HINDS).}

302. Marginella quinqueplicata (Lam.), Bay of Bengal, Malacca, Sumatra.

303. Marginella olivella (Reeve), N. S. Wales, Australia. Yucatan.

304. Marginella oblongata (Swainson), Bahamas and

305. Marginella guttata (Dillwyn). W. I., Florida, Beaufort, N. Carolina.

306. Marginella nivosa (Hinds), West Indies. 
hamas.

307. Marginella apicina (Menke), W. I., Florida, Ba-

308. Marginella pellucida (Pfeiffer), West Indies, Bahamas.

\section{SECTION VOLUTELLA (SWAINSON).}

309. Marginella bullata (Born), Bahia, Brazil.

310. Marginella angustata (Sowb.), Ind. O., Australia.

\section{SECTION PERSICULA (SCHUMACHER)}

3Ir. Marginella cornea (Lam.), West Africa. Is.

312. Marginella persicula (Linn.), Senegambia, C. Verde

313. Marginella cingulata (Dillwyn), W. Africa, Canaries, C. Verde Is.

314. Marginella porcellana (Gmel.), Venzuela.

3r5. Marginella interrupte lineata (Muhlf.), Liberia, West Indies, Venezuela.

\section{SUB-GENUS VOLVARIA (LAM.)}

316. Marginella avena (Vaenc.), W. Indies.

368. Marginella avena V :- Beyerleana (Bernardi), West Indies.

\section{FAMILY OLIVIDAE.}

Sub-Family Olivinae, Genus Olivella (Swainson.)

3r7. Olivella Verreauxi (Ducros), W. I.

318. Olivella mutica (Say), North Carolina, W. I.

319. Olivella mutica V :- nitidula (Dillw.), South Carolina, Brazil.

320. Olivella nivea (Gmel.), Bahamas, W. I., Brazil.

321 . Olivella myriadina (Duclos.), Panama.

322. Olivella jaspidea (Gmel.), W. I.

323. Olivella floralia (Duclos.), W. I.

324. Olivella lepta (Duclos.), China Sea, Jap., Phil.

325. Olivella Anagora (Duclos), Xipixapi, W. Columbia, Acapulco, Mazatlan.

326. Olivellà puelchana (D’Orb), San Blas, Patagonia, Carthagena, Cen. Amer. 
328. Olivella gracilis (Brod. and Sowb.), Mazatlan, Lower California.

329. Olivella dama (Mawe), Guaymas, Mazatlan.

$$
\text { GENUS OLIVA (BRUG.) }
$$

Sub-Genus Lamprodoma (Swainson).

330. Oliva volutella (Lam.), Panama to Gulf of Cal.

GENUS OLIVA (TYPICAL).

331. Oliva porphyria (Linn.), Panama to Mazatlan.

327. Oliva splendidula (Sowb.), Panama.

332. Oliva Peruviana (Lam.), Peru and Chili.

333. Oliva guttata (Lam.), Ind. O., China, Phil., Poly., Madagascar, Zanzibar.

334. Oliva inflata (Lam.), Red Sea, Ceylon, E. Africa, Madagascar, Seychelles.

335. Oliva tigerina (Lam.), Ceylon, Java, Phil., E. Africa.

336. Oliva elegans (Lam.), Ind. O., Cen. Poly.

337. Oliva maura (Lam.), Indo Pacific and Austro Pacific provinces.

338. Oliva irisans (Lam.), Mauritius, Ceylon, Java, Phil., Austr., Poly.

339. Oliva irisans V:- erythrostoma (Lam.), Same localities.

340. Oliva irisans $\mathrm{V}$ :- textilina (Lam.), Same localities. L. Cal.

341. Oliva araneosa (Lam.), Panama to Cape St. Lucas,

342. Oliva angulata (Lam.), Panama to Guaymas.

343. Oliva reticularis (Lam.), Florida, W. I.

344. Oliva Duclosi (Reeve), China, Phil., Austr., New Zealand, Poly.

345. Oliva pygmea (Beeve), Habitat unknown.

346. Oliva ispidula (Linn.), Ind. O., Phil., Fiji Is., etc. 369. Oliva sidelia (Ducl.), China, Phil., New Guinea, Viti Is., Madagascar.

347. Oliva tessellata (Lam.), Maldives, Java, Phil., Austr., New Caledonia.

348. Oliva carneola (Gmel.), Java, Phil., New Caledonia, Cen. Poly. 
SUB-GENUS CALLIANAX (H.\& A. AD.)

349. Oliva biplicata (Sowb.), California. SUB-GENUS AGARONIA (GRAY) to Mazatlan.

351. Oliva acuminata (Lam.), Senegal, Gambia, Java, Phil., Austr.

352. Oliva nebulosa (Lam.), Ceylon, W. Africa, Austr?

353. Oliva gibbosa (Born.), Ceylon, W. Africa.

SUB-GENUS OLIVANCILLARIA (D'ORB.)

354. Oliva Brasiliana (Lam.), Brazil, La Plata, Patagonia. Africa.

355. Oliva auricularia (Lam.), Brazil to Patagonia, W.

\section{SUB-FAMILY ANCILLARIINAE}

Genus Ancillaria (Lamark) Gulf, Zanzibar.

356. Ancillaria cinnamonea (Lam.), Red Sea, Persian

357. Ancillaria australis (Sowb.), Austr., New Zealand, Tasmania, Cape.

358. Ancillaria cingulata (Sowb.), N. Australia, China?

370. Ancillaria marginata (Lam.), Austr., Tasmania.

SUB-GENUS AUOLACIA (GRAY)

359. Ancillaria Mauritiana (Sowb.), Madagascar, Mauritius, Australia.

\section{SUB-GENUS DIPSACCUS (KLEIN)}

36o. Ancillaria glabrata (Linn.), W. I.

\section{SUB-FAMILY HARPINAE}

Genus Harpa (LAM.)

371. Harpa costata (Linn.), Mauritius.

372. Harpa ventricosa (Lam.), Ind. O., Zanzibar, Mauritius, Phil., Viti Is.

373. Harpa conoidal is (Lam.), Ind. O., Phil., Mauritius.

374. Harpa crenata (Swains.), Acapulco, Panama.

375. Harpa rosea (Lam.), Guinea, Senegal.

376. Harpa nobilis (Lam.), Ind. O., Phil., Viti Is.

377. Harpa minor (Lam.), Ind. O., So. Africa, Madagascar, Viti. 


\section{FAMILY COLUMBELLIDAE}

Genus Columbella (Lamark), Section I, Columbella (Typical) 378. Columbella rustica (Linn.), W. I., W. Africa, Cape Verde Is., S. Europe, Med. S.

379. Columbella fulgurans (Lam.), Phil., Solomon's Is., N. E. Austr., New Guinea, etc.

SECTION II, NITIDELLA (SWAINS.)

380. Columbella laevigata (Linn.), West Indies.

SECTION III, CONIDEA (SWAINS.)

$3^{8 r}$. Columbella flava (Brug.), Ind. O, Jap., Mauritius, New Caledonia, Poly.

\section{GENUS ENGINA (GRAY)}

\section{Sub-Genus Fusiostoma (Swains.)}

$3^{8} 3$. Engina nodicostata (Pease). Paumotus Is., Vtti Is.

382. Engina mendicaria (Linn.), East Indies, Phil. Austr., Poly.

\section{FAMILY CONIDAE}

Genus Conus (Linn.), Section I, Marmorei.

384. Conus marmorevs (Linn.), Ind. O., Jap., Austr., Poly., etc.

385. Conus araneosus (Hwass.), Ceylon, Phil., Moluccas.

3 S6. Conus imperialis (Linn.), Ceylon, Java to N. Cal., and Viti Is.

\section{SECTION II, LITERATI.}

387. Conus literatus (Linn.), Zanzibar, Ceylon, Java Singapore, New Cal., Viti Is.

388. Conus literatus V:- millepunctatus (Lam.), Same localities.

389. Conus musicus (Hwass.), Phil., Austr., Solomon's Is., New Caledonia.

390. Conus tessellatus (Born), Red Sea, Ceylon, Mozambique, Maur., Phil., N. Cal., Poly.

39r. Conus Protevs (Hwass), W. I., Florida. duras.

392. Conus flammens (Lam.), W. C. of Africa, Hon-

393. Conus venulatus (Hwass), W. C. of Africa. Senegal.

394. Conus Prometheus (Hwass), E. Africa, Madagascar, 
SECTION III, FIGULINI.

395. Conus betulinus (Linn.), E. Africa, Is. of Bourbon, Ceylon, Java, China, Phil.

396. Conus figulinus (Linn.), Ceylon, Java, Phil., New Caledonia.

397. Conus figulinus V :- Loroisi (Kien.), Same localities.

398. Conus quercinus (Hwass), Red S., E. Africa, Mauritius, Ceylon, Phil., Sandw. Is.

\section{SECTION IV, ARENATI.}

399. Conus arenatus (Hwass.). Red Sea, E. Africa, Ceylon, Phil., N. Cal., Viti Is.

410. Conus obesus (Hwass.), Ceylon, Java.

400. Conus pulicarius (Hwass.), Japan, N. Guinea, N. Cal., Cen. Poly.

\section{SECTION V, MURES.}

401. Conus mus (Hwass.), W. I., Florida.

402. Conus Hebraeus (Linn.), Ceylon, E. Africa, Maur., Jap., Phil., N. Cal.. Viti Is.

403. Conus Hebraeus V :- vermiculatus (Hwass.), Same localities.

404. Conus miliaris (Hwass), Red Sea, Is. of Bourbon, Natal, Sandw. and Galapagos Is.

405. Conus baccatus? (Sowb.), ?

406. Conus Ceylonensis (Hwass.), Red S., Ceylon, W. Africa, Maur., Austr., Poly., Mazatlan.

407. Conus Ceylonensis V :- sponsalis, Phil., N. Caledonia, Poly.

\section{SECTION VI. VARII}

40S. Conus gladiator (Brod.), W. C. of Cen. Amer.

409. Conus princeps (Linn.), W. C. of Cen. Amer.

SECTION VII, ADMIRALES

4II. Conus articulatus (Sowb.), Mauritius.

4I2. Conus Macarae (Bernardi), Mauritius.

413. Conus generalis (Linn.), Red S., Ceylon, Is. of Bourbon, E. Africa, E. I., Phil.

\section{SECTION VIII, CAPITANEI}

4I4. Conus vexillum (Gmelin.), E. Africa, Ceylon, Java, Phil., N. Cal., Samoan Is. 
415. Conus miles (Linn.), E. Africa, Ceylon, Jap., Phil . N. Cal., Cen. Poly.

416. Conus vittatus (Lam.), Panama, Real Llejos.

\section{SECTION IX, VIRGINES.}

4r7. Conus Virgo (Linn.), Red Sea, E. Africa, Ceylon, Phil., N. Cal., Poly.

418. Conus flavidus (Lam.), Red Sea, E. Africa, Ceylon, Java, Austr., N. Cal., Poly.

\section{SECTION X, DAVCI.}

419. Conus lineatus (Chemn.) [Auct.], Red S., E. Africa, Ceylon, Phil., Austr., N. Cal.

\section{SECTION XI, MAGI}

420. Conus magus (Linn.), Madagascar, Borneo, Austr., N. Cal., Phil., etc.

421. Conus mercator (Linn.), W. I., W. Africa. etc.

422. Conus spectrum (Linn.), Maur., Java., Moluccas,

423. Conus interruptus (Brod.), W. C. of Cen. Amer., Mazatlan, Cape St. Lucas.

\section{SECTION XII, ACHATINI.}

424. Conus catus (Hwass.), Red S., Maur., Java, China, N. Caledonia, Poly.

425. Conus purpurascens (Brod.), Panama to Mazatlan.

426. Conus Guinaicus (Hwass.), W. C. of Africa.

SECTION XIII, ASPERI. luccas.

427. Conus australis (Chenm.), [Auct•], Australia, Mo-

428. Conus sulcatus (Hwass.), China, Singapore.

429. Conus verrucosus (Hwass.), W. C. of Africa, W. I. SECTION XIV, GLANS.

430. Conus glans (Hwass.), Phil., Aust.. N. Cal., Poly. Annaa.

431. Conus tendinens (Hwass.), I. Bourbon, Mauritius,

432. Conus nussatella (Linn.), Red S., E. Africa, Ceylon, Java, Phil., N. Austr., Poly. 
SECTION XVI, TULIPAE.

435. Conus striatus (Linn.), Red S., E. Africa, Ceylon, Phil., Austr., N. Cal., Viti Is.

433. Conus gubernator, E. Africa, Madagascar, Maur., Ceylon, Phil., N. Cal.

434. Conus rhododendron (Conthony) [Jay], Austr., N. Guinea, Poly.

436. Conus tulipa (Linn.), E. Africa, Red S., Ceylon, Phil., N. Cal., Poly.

437. Conus geographus (Linn.), E. Africa, Red S., Ceylon, Phil., N. Cal., Poly. ties.

438. Conus geographus $\mathrm{V}$ :- mappa (Crosse), same locali-

\section{SECTION XVII, TEXTI.}

439. Conus retifer (Menke), Phil., Sandw., Is.

440. Conus textile (Linn.), Maur., Red S., Ceylon, Jap., Phil., Austr., Viti Is.

441. Conus textile $V$ :- telatus (Recve), same localities.

442. Conus textile $V$ :- vericulum (Reeve), same localities. localities.

443. Conus textile V:- complanatus (Sowb.), same

444. Conus lucidus (Mawe), Isle of La Plata, W. C. of Cen. America.

445. Conus omaria V :- colubrinus (Lam.), Red S, Ceylon, Phil., Austr., Poly.

446. Conus aulicus (Linn.), Maur., Ceylon, Phil.. N. Cal., Viti Is., etc.

\section{FAMILY PLEUROTOMIDAE.}

Sub-Family Pleurotominae. Genus Pleurotoma (Lamark)

447. Pleurotoma babylonia (Linn.), Phil., Moluccas, New Guinea, Timor, Maur.

448. Pleurotoma Garmonsii (Reeve), Red S., E. Africa, Mauritius, Java.

449. Pleurotoma tigrina (Lamark), Phil., Viti Is.

450. Pleurotoma albina (Lam.), Moluccas.

45 I. Pleurotoma gracillima (Weinkauff.), ?

452. Pleurotoma cingulifera (Lam.), Red S., Phil., Sandw. Is.

453. Pleurotoma Indica (Desh.), China. 
GENUS GENOTIA (H. \& A. ADAMS.)

454. Genotia Mitraeformis (Wood), W. Africa. SUB-FAMILY CLAVATULINAE.

Genus Clavatula (Lam.) Africa.

455. Clavatula muricata (Lam.), W. C. of Africa, S.

\section{GENUS PUSIONELLA (GRAY)} Algiers.

456. Pusionella Nifat (Adamson) [Bruguiere], W. Africa,

GENUS SURCULA (H. \& A. AD.) California.

457. Surcula maculosa (Sowb.), W. Columbia, G. of

458. Surcula australis (Roissy), China S., Philippines.

FAMILY TEREBRIDAE.

Genus Terebra (Adamson)

459. Terebra crenulata (Linn.), Ind. O., Cent. Poly. Poly.

460. Terebra dimidiata (Linn.), Singapore, Phil., Cent.

46r. Terebra maculata (Linn.), Moluccas, Austr., Phil., Cent. Poly.

462. Terebra muscaria (Lam.)' Japan, Phil.. Cent. Poly. 463 Terebra subulata (Linn.), Japan, Moluccas, Phil., Society Is., etc.

464. Terebra oculata (Lam.), Moluccas, Viti Is.

465. Terebra chlorata (Lam.), Malacca, Seychelles, Mauritius, Sandw., Is.

466. Terebra strigata (Sowb.), Panama, Realejo.

467. Terebra cerethina (Lam.), Phil., Society Is.

468. Terebra lanceata (Linn.), Taheiti, Moluccas, Mauritius.

469. Terebra affinis (Gray), Phil., Seychelles, Viti Is.

470. Terebra variegata (Gray), Mazatlan, Guaymas, S. California, China S., W. Africa.

47 I. Terebra concinnia (Desh.), Sandwich Is.

472. Terebra Dussumieri (Kien), China, Japan.

473. Terebra duplicata (Linn.), Moluccas, Singapore, Zanzibar, China, Viti Is. 
474. Terebra duplicata V :- Lamarkii (Kien.), same localities.

475. Terebra longiscata (Desh.), Phil.

476. Terebra dislocata (Say), North Carolina, W. I.

477. Terebra decorata (Desh.), Pidang, Sumatra.

478. Terebra cingulifera (Lam.), Phil., New Ireland, Viti Is., China.

479. Terebra caerulescens (Lam.), Austr., Phil., Poly., Maur., Red S.

480. Terebra aciculina (Reeve), Singapore, Manilla, Marquesas, Sandw. Is.

48r. Terebra strigulata (Linn.), Poly., Sandw. Is.

482. Terebra brastata (Gmel.), W. I., W. Africa.

FAMILY CANCELLARIA.

Genus Cancellaria (Lam.), Section Cancellaria.

483. Cancellaria reticularia (Linn.), W. I., Florida.

SECTION TRIGONOSTOMA (BLAINV.)

484. Cancellaria goniostoma (Sowb.), W. C. of Cen. America, Mazatlan.

485. Cancellaria scalarina (Lam.), Isle of France.

486. Cancellaria obliquata (Lam.), Phil., Zanzibar.

FAMILY STROMBIDAE.

Sub-Family Strombinae, Genus Strombus (L.), Section I, Strombus.

487. Strombus costatus (Gmel.), West Indies.

Is.

488. Strombus bubonius (Lam.), Senegal and Cape Verde Brazil.

489. Strombus pugilis (Linn.) So. Carolina, W. I.

490. Strombus pugilis, V :- alatus (Gmel.), same localities.

491. Strombus gracilior (Sowb.), Panama, Mazatlan, La Paz, California.

492. Strombus canarium (Linn.), Red S., Ind. O., Phil. ities.

493. Strombus canarium V :- Isabella (Lam.), same local-

494. Strombus granulatus (Gray), Panama, Mazatlan, Galapagos Is. 


\section{SECTION II, EUPROTOMUS (GILL)}

495. Strombus lentiginosus (Linn.), Phil., N. Caledonia, zibar.

496. Strombus papilio (Chemn.)[Dillwyn.], Phil, Zan-

497. Strombus laciniatus (Chemn.)[Dillw.], Moluccas, Phil., N. Caledonia, Viti Is. Viti Is.

498. Strombus latissimus (Linn.), Amboina, Japan, Phil.,

499. Strombus ponderosus (Phil.), New Caledonia, Society Is.

\section{SECTION III, MONODACTYLUS (KLEIN)}

500. Strombus Peruvians (Swains), Peru, W. Columbia.

50r. Strombus tricornis (Lam.), Red S., Isle of Bourbon, Seychelles, Phil.

502. Strombus bituberculatus (Lam.), W. I., N. South America.

503. Strombus gallus (Linn.), West Indies.

504. Strombus auris-Dianae (Linn.), Ind. O., Maur., Phil., Society Is.

505. Strombus auris-Dianae, V :- melanostoma (Swains), same localities. Society Is.

506. Strombus Pacificus (Swainson), Japan, Australia, SECTION IV, GALLINULA (KLEIN.)

507. Strombus vittatus (Linn.), Ind. O., China, Phil.

508. Strombus Japonicus (Reeve), Japan.

509. Strombus columba (Lam.), Red S., Zanzibar, Phil., Pelew. Is. donia.

510. Strombus epidromis (Linn.), China, Phil., N: Cale-

5Ir. Strombus succinctus (Linn.), Ind. O., Phil.

5I2. Strombus variabilis (Swains.), Zanzibar, Phil., Austr., Samoa.

513. Strombus minimus (Linn.), Phil., Viti Is. SECTION V, CANARIUM (SCHUM.)

514. Strombus urceus (Linn.), Ind. O., Phil., Austr. 
515. Strombus dentatus (Linn.), Red S., Phil., New Caledonia, Viti Is., Mauritius. Viti Is.

516. Strombus floridus (Lam.), Zanzibar, Jap., Austr.,

517. Strombus faciatus (Born.), Red S., Ind. O., Phil.?

518. Strombus maculatus (Nutall.), Sandw., Paumotus, and Friendly Is.

519. Strombus gibberulus (Linn.), Zanzibar, Natal, Red S., Ind. O., Maur., Phil., Viti Is., etc.

520. Strombus Samar (Chemn.) [Dillw.], Phil., Austr., Society and Paumotus Is.

\section{SECTION VI, CONOMUREX (BAYLE).} etc.

521. Strombus Luhuanus (Linn.), Phil., Austr., Viti Is.,

\section{GENUS PTEROCERA (LAM.)}

Section I, Pterocera, Sub-Section I, Heptadactylus (Klein.)

522. Pterocera lambis (Linn.), Ind. O., Zanzibar, Phil., New Guinea, Society Is., etc.

523. Pterocera aurantia (Lam.), Phil., Mauritius, etc. SUB-SECTION II, MILLIPES (KLEIN)

524. Pterocera scorpio (Linn.), Ind. O., Phil.

525. Pterocera millepeda (Linn.), Ind. O., Maur., N. Guinea, Phil.

526. Pterocera elongata (Swains), Ind. O.

\section{SECTION II, HARPAGO (KLEIN)}

527. Pterocera auragra (Linn.), Ind. O., Phil., N. Caledonia, Poly. etc.

528. Pterocera rugosa (Sowb.), Zanzibar, Society Is.,

GENUS ROSTELLARIA (LAM.)

-529. Rostellaria curvirostris (Lam.), Red S., Moluccas.

530. Rostellaria fusus (Linn.), China.

\section{GENUS TEREBELLUM (KLEIN.)}

531. Terebellum subulatum (Lam.), Ind. O., Java, China S., Phil., Viti Is., Maur. 
SUB-FAMILY APORRHAINAE.

Genus Aporrhais (Dillw.), Section I, Aporrhais. Jceland.

532. Aporrhais pes pelecani (Linn.), Seas of Europe,

SUB-FAMILY STRUTHIOLARIINAE.

Genus Struthiolaria (Lam.), Section I, Struthiolaria.

533. Struthiolaria papulosa (Martyn.), New Zealand.

534. Struthiolaria vermis (Mart.), New Zealand.

535. Struthiolaria gigas (Sowb.), New Zealand.

536. Young of Strombus pugilis? See 489

\section{FAMILY CYPRAEIDAE}

Genus Cypraea (Linn.)

Hebrides.

538. Cypraea cervus (Linn.), Panama.

Mazatlan.

539. Cypraea exanthema (Linn.), W. I., Flor., Panama,

540. Cypraea scurra (Chemn.), Annaa Is., Austr.

541. Cypraea testudinaria (Linn.), Ceylon, N. Caledonia.

542. Cypraea Isabella (Linn.), Ind, and Pac. O.

543. Cypraea lurida (Linn.), Med. S.

544. Cypraea cinerea (Gmel.), W. I.

545. Cypraea carneola (Linn.), Pac. and Ind. O.

546. Cypraea talpa (Linn), Pac. and Ind. O.

547. Cypraea interrupta (Gray), Ceylon.

548. Cypraea quadrimaculata (Gray), Borneo, Ind. O.

549. Cypraea irrorata (Sol.), Elizabeth Is., Paumotus. Maur.

549 I-2 Cypraea microdon (Gray), (Loyalty Is.), Phil.,

550. Cypraea felina ( $\mathrm{Gmel}$ ), Ind. and Pac. O.

$55^{\mathrm{I}}$. Cypraea felina $\mathrm{V}$ :- urcellus (Gmel.), Same localities. O.

552. Cypraea hirundo (Linn.), New Cale., Austr.. Ind. Cale.

553. Cypraea cylindrica (Born.), Ceylon, Austr., N.

554. Cypraea tabescens (Sol.), N. Cale., Loyalty Is., Austr.

555. Cypraea cruenta (Gmel.), Ind. O., N. S. Wales. 
N. Cale.

556. Cypraea stolida (Linn.), Ceylon, Borneo, Sandw. Is.

557. Cypraea Erythraeensis (Beck.), Red S.

558. Cypraea ventriculus (Lam.),Annaa Is., Pacific O.

559. Cypraea arenosa (Gray), Annaa Is., Cen. Pac.

560. Cypraea sulcidentata (Gray), Austr., South Seas, Sandw. Is. Is.?

561. Cypraea tessellata (Swn.), New Zealand, Sandw.

562. Cypraea Mauritiana (Linn.), Samoa, N. Cale., Borneo, Ceylon.

563. Cypraea caput-serpentis (Linn.), Ind. and Pac. O.

564. Cypraea mappa (Linn.), N. Cale., Java, Ind. O.

Ind. $\mathrm{O}$.

565. Cypraea Arabica (Linn.), Samoa, N. Cale., Austr.,

566. Cypraea reticulata (Martyn.), Ind. and Pac. O.

567. Cypraea reticulata V:- intermedia (Gray), Same

568. Cypraea histrio (Mensch.), Tonga Tabou, Ind. O.

569. Cypraea arabicula (Lam.), Acapulco, G. of Cal.

570. Cypraea stercoraria (Linn.), W. Africa.

571. Cypraea Scotti (Brod.), W. Austr.

572. Cypraea thersites (Gask.), S. Austr.

573. Cypraea usus (Linn.), At. O., Med. S. Taheiti, Japan.

574. Cypraea moneta (Linn.), Maldive Is., Austr., ties.

575. Cypraea moneta V :- icterina (Lam.), Same locali-

576. Cypraea annulus (Linn.), Ind. and Pac. O.

577. Cypraea obvelata (Lam.), N. Cale., Samoa, Austr.

578. Cypraea tigris (Linn.), Ind. and Pac. O.

579. Cypraea pantherina (Soland.), Red S.

580. Cypraea umbilica (Sowb.), N. S. Wales.

$5^{8}$ r. Cypraea vitellus (Linn.), Ind. O., Austr., N. Cale. 581 I-2 Cypraea spadicea (Swn.), (Santa Barbara) San Diego, Cal.

582. Cypraea onyx (Linn.), Ceylon, Jap., Phil. Is.

583. Cypraea pyrum (Gmel.), Med. S., At. C. of S. Europe and Africa. 
Red S.

584. Cypraea lynx (Linn.), Ind. O., Austr., N. Cale., Ind. $\mathrm{O}$.

585. Cypraea errones (Linn.), China, Austr., N. Cale.,

586. Cypraea Sophiae (Brazier), San Christoval Is.

587. Cypraea subviridis (Reeve), Austr., N. Cale.

588. Cypraea pulchella (Swn.), China.

589. Cypraea nigro punctata (Gray), Galapagos Is.

590. Cypraea Sowerbyi (Kien.), Gulf of California.

591. Cypraea picta (Gray), Gambia, C. Verd Is.

592. Cypraea sanguinolenta (Gmel.), Gambia.

593. Cypraea undata (Lam.), Phil., Maur.

594. Cypraea ziczac (Linn.), Mozambique, Ceylon.

595. Cypraea asellus (Linn.), Austr., Samoa, Red S.

596. Cypraca clandestina (Linn.), Ceylon, Jap., Austr.

Borneo.

597. Cypraea punctata (Linn.), Is. of Bourbon, Phil.,

598. Cypraea piperita (Soland.), Austr.

599. Cypraea cribraria (Linn.), Ceylon, N. Cale., N. Ireland.

6oo. Cypraea cribellum (Gask.), Mauritius.

601. Cypraea Lamarki (Gray), Ind. and Pac. O.

6oI I-2 Cypraea miliaris (Gmel.), (Japan), N. S. Wales.

602. Cypraea eburnea (Barnes), Phil.

603. Cypraea turdus (Lam.), Gulf of Cal.

604. Cypraea erosa (Linn.), Ind. and Pac. O.

6ò. Cypraea ocellata (Linn.), Ceylon.

6o6. Cypraea Listeri (Gray), Phil.

607. Cypraea poraria (Linn.), Fiji Is., Austr., N. Cale.

6o8. Cypraea albuginosa (Mawe), Gulf of Cal.

609. Cypraea helvola (Linn.), Ind. and Pac. O. Austr.

6ro. Cypraea citrina (Gray), Ceylon, Maur., N. W.

6ro I-2 Cypraea semiplota (Mighels), Oahu.

6ri. Cypraea spurca (Linn.), Med. S., E. At. O. GROUP GASKONIA (ROBERTS)

6I2. Cypraea edentula (Sowb.), South Africa. 
GROUP PUSTULARIA (SWN.)

613. Cypraea staphylaea (Linn.), Ind. and Pac. O.

6i4. Cypraea pustulata (Lam.), Panama, Mazatlan.

6i 5. Cypraea nucleus (Linn.), Ind. and Pac. O.

6I6. Cypraea Madagascariensis (Gmel.), Madagascar, Sandw. Is.

SECTION EPONA (H. \& A. AD.)

6r7. Cypraea annulata (Gray), Maur., Phil.

6r8. Cypraea cicercula (Linn.), Maur., Borneo, W. Cale.

6ig. Cypraea globulus (Linn.), Maur., Borneo, N. Cale. Cale.

620. Cypraea Childreni (Gray), Annaa Is., Borneo, N.

\section{SUB-GENUS TRIVIA (GRAY)}

621. Trivia costata (G mel.), W. I., C. of G. H. ?

622. Trivia vitrea (Gask.), Phil.

623. Trivia pediculus (Linn.), Flor., W.I.

623 I-2 Trivia suffusa (Gray), (West Indies.)

624. Trivia quadripunctata (Gray), W. I., Florida.

625. Trivia radians (Lam.), L. California, (Ecuador).

625 I-2 Trivia Solandri (Gray), (Acapulco), Santa Barbara.

626. Trivia pellucidula (Gask.), Pac. O.

627. Trivia brevissima (Sowb.), ?

628. Trivia Europaea (Montagu), Med. S., Norway.

629. Trivia Australis (Lam.), N. S. Wales.

63o. Trivia pulex (Soland.), Azores, Med. S.

631. Young Cypraeae.

\section{FAMILY OVULIDAE.}

\section{Genus Ovula (Brug.)}

632. Ovula ovum (Linn.), E. Africa, Ind. O., Jap., Society Is.

633. Ovula tortilis (Martyn), Zanzibar, Moluccas, Friendly Is.

634. Ovula pyrulina (A. AD.), New Caledonia. SUB-GENUS CYPHOMA (BOLTEN)

635. Ovula gibbosa (Linn.), Flor., W. I. 


\section{SUB-GENUS VOLVA (BOLTEN.)}

636. Ovula volva (Linn.), China, Phil., Jap. SUB-GENUS CALPURNUS (MONTF.)

N. Cale.

68I. Ovula acicularis (Lam.), W. I., S. Carolina, Florida.

\section{FAMILY DOLIIDAE.}

\section{Genus Dolium (Lam.)}

638. Dolium galea (Linn.), Med. S., Beaufort, N. C., W. I., Brazil.

639. Dolium variegatum (Lam.), North Australia.

640. Dolium olearium (Brug.), Ind. O., Phil.

641. Dolium zonatum (Green), China, Japan.

642. Dolium faciatum (Brug.), Phil., China, Japan.

643. Dolium costatum (Menke.), E. Indies, Phil., Maur. localities.

644. Dolium costatum V:- maculatum (Lam.), same

645. Dolium perdix (Linn.), Ind. O., Maur., Poly., W. Africa, W. I., Brazil.

\section{SUB-GENUS MALEA (VAL.)}

646. Dolium pomum (Linn.), Red S., Ind. O., Phil., Society Is.

647. Dolium ringens (Swains.), Peru, Panama, Mazatlan. GENUS PYRULA (LAM.)

648. Pyrula reticula (Lam.), Ind. O. E. Indies, Jap.

649. Pyrula decussata (Wood), Panama to Cape St. Lucas, L. California.

65o. Pyrula papyratia (Say.), Beaufort, N. C., W. I.

651. Pyrula ficus (Linn.), Red S., Ind. O., Singapore.

652. Pyrula Dussumieri (Valenc.), China.

653. Pyrula tessellata (Kobelt.), Rosemary Is.. Austr.

FAMILY CASSIDIDAE.

Genus Cassis (Klein.)

654. Cassis cornuta (Linn.), Ind. O., Tranquebar, Maur., Jap., Phil., W. I. 
655. Cassis cameo (Stimpson), Beaufort, N. C., W. I., Brazil.

656. Cassis tuberosa (Linn.), Beaufort, N. C., W. I.,

657. Cassis flammea (Linn.), W. I.

658. Cassis spinosa (Gronov.), W. Africa, Austr.

659. Cassis fimbriata (Quoy), Austr.

\section{SECTION III, CYPRAECASSIS (STUTCHBURY)}

66o. Cassis tenuis (Gray), Galapagos Is.

66r. Cassis testiculus (Linn.), W. I.

662. Cassis crumena (Brug.), W. Africa, Cape Verde Is. W. I.

663. Cassis rufa (Linn.), Ind. O., Maur., Jap. SUB-GENUS SEMICASSIS (KLEIN.)

664. Cassis sulcosa (Brug.), Med. S, Portugal, W. C. of Africa, W. I., Brazil, Panama, Guaymas.

665. Cassis sulcosa V :- inflata (Shaw), Same Localities.

666. Cassis sulcosa V:- abbreviata (Lam.), Same Localities.

667. Cassis saburon (Adamson), Med.S., Spain, Senegal.

SECTION II, BEZOARDICA (SCHUM.)

668. Cassis glauca (Linn.), Singapore, Phil.

669. Cassis coronulata (Sowerby.), Jap., Phil., Austr.

67o. Cassis exarata (Reeve), Is. of Bourbon.

671. Cassis areola (Linn.), Ind. O., Phil., N. S. Wales.

672. Cassis strigata (Gmel.), Moluccas, China, Jap., Phil.

SECTION III, CASMARIA (H. \& A. AD.)

673. Cassis vibex (Linn.), Maur., Phil., Society Is., Paumotus.

674. Cassis vibex V :- erinacea, Same Localities.

675. Cassis torquata (Reeve.), Port Jackson, Austr.

676. Cassis achatina (Lam.), C. of G. H., Maur., Austr., N. Zealand, Phil.

677. Cassis achatina (Lam. V :- pyrum), Austr., Maur., Natal, C. Verde Is. 


\section{GENUS CASSIDARIA (LAM.)}

678. Cassidaria echinophora (Linn.), Med. S.

679. Cassidaria Tyrrhena (Lam.), Med. S., At. C. of France and Spain.

\section{GENUS ONISCIA (SOWB.)}

680. Oniscia oniscus (Linn.), W. I. SECTION ONISCIDIA (SWAINS.)

68r. Oniscia cancellata (Sowb.), China.

\section{FAMILY NATICIDAE.}

Sub-Family Naticinae, Genus Natica (Adamson), Sub-Genus Natica.

682. Natica millepunctata (Lamark), Med. Sea.

683. Natica Chinensis (Lamark), China, Singapore, Phil., Viti Is. Florida.

684. Natica canrena (Linn.), W. I., Cen. America,

685. Natica marochiensis (Gmelin), W. Africa, W. I., Panama, Mazatlan, Phil., Australia.

686. Natica collaria (Lamark), West Africa.

687. Natica lineata (Lamark), Singapore, Phil. SUB-GENUS NEVERTIA (RISSO.)

\section{Section Nevertia.}

688. Natica duplicata (Say.), New Eng., Florida, Gulf Shores of the U. S. Mexico.

689. Natica Recluziana (Desh.), Cal., N. W. Coast of

\section{SECTION MANIMA (KLEIN.)}

690. Natica aurantia (Lamark), Singapore, Moluccas, Phil., Austr., New Caledonia.

691. Natica ravida (Souleyet), Payta, Peru, St. Helena, W. Columbia.

692. Natica orientalis (Gmelin), Singapore.

693. Natica melastoma (Swainson), Australia.

694. Natica solida (Blainville), Singapore, Phil.

695. Natica mamilla (Linn.), East Indies, Phil., New Caledonia, Cen. Poly. 


\section{ISECTION MAMILLA (SCHUM.)}

696. Natica melanostoma (Gmel.), E. I., Phil., W. Poly., Maur., Madagascar.

697. Natica maura (Brug.), Phil.

SUB-GENUS AMPULLINA (LAM.), DEFRANCE.

698. Natica fluctuata (Sowb.), Phil.

GENUS SIGARETUS (LAM.)

699. Sigaretus Martinianus, W. I.

700. Sigaretus perspectivus (Say-), New Jersey (rare), N. Carolina, W.I.

FAMILY CALYPTRAEIDAE.

Sub-Family Calyptraeinae, Genus Crucibulum (Schum.)

7or. Crucibulum scutellatum (Gray), Chili, Mazatlan.

GENUS CALYPTRAEA (LAMARK)

Sub-Genus Infundibulum (Montfort).

702. Calyptraea radians (Lamark), Peru, Chili.

GENUS CREPIDULA (LAMARK) Brazil.

703. Crepidula fornicata (Linn.), Maine, Florida, W. I.,

\section{- SEstí SECTION IANACUS (MORCH.)}

704. Crepidula unguiformis (Lamark), Nova Scotia, Texas, W. I., Med. Sea, Peru, Austr., Panama, Cal.

GENUS CAPULUS (MONTFORT)

705. Capulus Hungaricus (Linn.), Europe, Martha's Vineyard Sound.

\section{SUB-FAMILY HIPPONYCINAE.}

Genus Hipponyx (Defrancr).

706. Hipponyx antiquatus (Linn.), Flor., W. I., Poly., Austr., Calif., Peru, Senegal, Panama.

\section{GENUS MITRULARIA (SCHUM.)}

707. Mitrularia equestris (Linn.), China Sea, Phil., Ind. O., W. Africa, W. I., W. coast of Cen. Am.

FAMILY XENOPHORIDAE.

Genus Xenophora (Fischer de Waldheim).

708. Xenophora conchyliophorus (Born:), West Indies. 
SECTION TUGURIUM (FISCHER).

709. Xenophora exerta (Reeve), China.

710. Xenophora helvacea (Phil.), Madagascar.

SECTION ONUSTUS (H. \& A. AD.)

711. Xenophora solaris (Linn.), Malacca, Singapore.

FAMILY VERMETIDAE.

Genus Vermetus (Adamson), Sub-Genus Vermicularia (Lam.)

712. Vermetus lumbricalis (L.), Phil., East Indies.

713. Vermetus spiratus (Phil.), At. Coast of the U.S., W. I., Gulf of Mexico.

\section{GENUS SILIQUARIA (BRUG.)}

Section Siliquaria.

7r4. Siliquaria obtusa (Schum.), Med. Sea.

715. Unidentified species of Vermetus.

FAMILY TURRITELLIDAE.

Genus Turritella (Lam.), Section Turritella.

716. Turritella terebra (L.), Singapore, Java, Phil.

SECTION HANSTATOR (MONTFORT). lan.

718. Turritella goniostoma (Val.), Payta, Peru, Mazat-

719. Turritella variegata (Linn.), W. I.

720. Turritella cingulata (Sowb.), Chili.

721. Turritella bicingulata (Lam.), Cape Verde Is.

SECTION ZARIA (GRAY)

722. Turritella duplicata (Linn.), Ind. Ocean.

ADDENDA.

Section Turritellopsis (Sars.)

722 I-2 Turritella reticulata (Mighels \& Adams).

SUB-GENUS MESALIA (GRAY)

722 3-4 Turritella brevialis (Lam.), N. \& W. Africa.

FAMILY PYRAMIDELLIDAE.

Genus Pyramidella (Lam.), Sub-Genus Pyramidella.

723. Pyramidella dolabrata (L.), W. I. 


\section{SECTION LONCHAEUS (MORCH.)}

724. Pyramidella acus (Gmel.), Poly, Maur, Red Sea.

FAMILY SOLARIIDAE.

Sub-Family Solariinae, Genus Solarium (Lam.), Sub-Genus Solarium (Lam.)

725. Solarium perspectivum (Linn.), Ind O., Pac. O., China, Austr.

726. Solarium modestum (Phil.), China, Society Is. SUB-FAMILY TORINIINAE.

Genus Torinia (Gray), Sub-Genus Torinia. O.

726 I-2 Torinia variegata (Gmel.), Red S., Ind. O., Pac.

\section{FAMILY IANTHINIDAE.}

Genus Ianthina (Lam.). Pac. O.

727. Ianthina fragilis (Lam.), Med., At. O., Ind. O., FAMILY SCALARIIDAE.

Genus Scalaria (Lam.), Section Scalaria.

728. Scalaria pretiosa (Lam.), China, Austr., Moluccas. SECTION CLATHRUS (OKEN).

729. Scalaria communis (Lam.), Europe. SECTION OPALIA (H. \& A. AD.). Maur.

730. Scalaria lamellosa (Lam.), Med., W. I., Sandw. Is.,

\section{SECTION CIRSOTREMA (MORCH)}

731. Scalaria varicosa (Lam.), Phil., Austr., Maur. FAMILY CERITHIIDAE.

Genus Cerithium (Adamson), Sub-Genus Cerithium. Phil.

732. Cerithium nodulosum (Brug.), Singapore, Moluccas,

733. Cerithium adustum (Kien.), Gallapagos Is., Panama, Mazatlan.

734. Cerithium eburneum (Brug.), Flor., W. I. SUB-GENUS VERTAGUS (KLEIN.)

735. Cerithium aluco (Linn.), Phil., Viti Is. 
Sub-Genus Cerithium.

734 I-2 Cerithium muscarum (Say.), Florida, Bahamas.

734 3-4 Cerithium ustum (Hombr. et Jacq.), Solomon's Archipelago. tius.

736. Cerithium obeliscus (Brug.), E. Ind., Poly., Mauri-

737. Cerithium Kochii (Phil.), E. coast of Africa.

738. Cerithium asper (Linn.), Singapore, Maur., Madagascar, Friendly: Is.

739. Cerithium faciatum (Brug.), Ind. O,, Phil. $\mathrm{O}$.

740. Cerithium Pharos (Hinds), Paumotus, Viti Is., Ind.

741. Cerithium vertagus (Linn.), East Indies, Phil.

GENUS BITTIUM (LEACH) [GRAY]

74I I-2 Bittium lacteum (Phil.), Med. S., Canary Is. GENUS POTAMIDES (BROVG.)

Sub-Genus Pyrazus (Montfort).

742. Potamides ebeninus (Brug.), Austr., New Zealand.

SUB-GENUS TYMPANOTONOS (KLIEN) Africa.

743. Potamides fuscatus (L.), Gambia, Cape Palmas, W.

744. Potamides radula (Linn.), W. Africa.

745. Potamides fluviatilis (Potiez et Michayd), Ind. O., Singapore, Borneo, China, Jap., Phil., Austr.

746. Potamides palustris (L.), India, Java, Austr.

SUB-GENUS TEREBRALIA (SWAINS.) Phil.

747. Potamides sulcatus (Born.), Singapore, China,

SUB-GENUS TELESCOPIUM (MONTF.)

748. Potamides telescopium (L.), India, Singapore, Phil., Borneo.

\section{SUB-GENUS CERITHIDEA (SWAINS.)}

749. Potamides decollata (L.), India, Timor, Madagascar.

749 I -2 Potamides scalariformis (Say.), Florida. Belize.

749 3-4 Potamides iostoma (Pfr.), So. Carolina, W. I., 
FAMILY LITTORINIDAE.

Genus Littorina (Ferrusac.), Section Littorina.

750. Littorina littorea (L.), Europe, N. coast of the U.S.

751. Littorina rudis (Donovan), N. Europe, and U. S.

SECTION MELARAPHE (MUHLF.)

752. Littorina scabra (L.), Mazatlan, Sandw. Is., Viti Is., Phil., China, Ind. O.

753. Littorina varia (Sowb.), Peru, Panama, Mazatlan.

753 I-2 Littorina minima (Gray), W. I.

GENUS TECTARIUS (VALENCE)

753 3-4 Tectarius pagodus (Linn.), Australia, Moluccas, Ceylon, Zanzibar.

754. Tectarius bullatus (Martyn), Ind. O., Austr., Poly.

755. Tectarius muricatus (L.), W. I., Bahamas, Florida.

755 I-2 Tectarius nodulosus (Gmel.), N. Carolina, Bahamas, W. I., Ceylon, Phil., Austr., New Zealand.

GENUS MODULUS (GRAY)

756. Modulus tectum (Gmel.), Sandw. and Viti Is., Ind. O., Red Sea, Maur.

$75^{6}$ I-2 Modulus lenticularis (Chemn.) [Auct.], W. I.

\section{FAMILY PLANAXIDAE}

Sub-Family Planaxinae, Genus Planaxis (Lamark)

757. Planaxis sulcatus (Born.), Sandw. I., Austr., Phil., Maur., S. Africa.

758. Planaxis planicostatus (Sowb.), San Blas, Panama, Galapagos Is.

759. Planaxis nucleus (Lam.), W. I.

\section{FAMILY NERITIDAE}

Genus Nerita (Adamson), Section Nerita (Lam.)

76o. Nerita albicilla (Linn.), Red S., Ind. O., Natal, Singapore, China, Phil., Viti Is.

761. Nerita plexa (Chemn.), East Africa, Capr., Madagascar, India.

762. Nerita exuvia (Linn.), Ind. O., E. Indies, Phil.

763. Nerita reticulata (Karsten), East Indies, Poly. Is.

764. Nerita Senegalensis (Gmel.) W. Africa, Cape Verde 


\section{SECTION PELORONTA (OKEN)}

765. Nerita peloronta (Linn.), Florida, W. I.

766. Nerita versicolor (Gmel.), Florida, W. I.

SECTION PILA (KLEIN)

Sand. Is. Nerita plicata (Linn.), Ind. O., Formosa, Poly.,

768. Nerita grossa (Linn.), Ind. O., Phil., N. E. Austr.

769. Nerita undata (Linn.), East Ind., Phil.

770. Nerita scabricosta (Lam.), Panama, Lower Calif.

\section{SECTION ODONTOSTOMA (KLEIN) [MORCH]} Maur.

771. Nerita polita (Linn.), Red S., Ind. O., Phil., Poly.,

772. Nerita affinis (Reeve) Viti Is.

GENUS NERITINA (LAMARK)

Section Neritina (Swainson)

Bourbon.

773. Neritina gagates (Lam.), Maur., Seychelles, Isle of

774. Neritina ziczac (Sowb.), E. Indies, Poly.

775. Neritina zebra (Brug.), Northern So. America, Brazil, Porto Rico? Panama?

776. Neritina Smithi (Sowb.), Bengal.

777. Neritina turrita (Chemn.), E. Ind., Phil., Austr.

778. Neritina Turtoni (Recluz.), Viti Is. Phil.

779. Neritina communis (Quoy and Gaimard), E. Indies,

780. Neritina reclivata (Say.), Florida, W. I., Mexico. Cen. America.

78 r. Neritina virginea (Linn.), W. I., Brazil.

782. Neritina picta (Sowb.), Gulf of Calif., Panama.

783. Neritina Nalanensis (Lesson), Ind. O., Poly.

SECTION PUPERITA (GRAY)

784. Neritina pupa (Linn.), W. I.

SECTION NERITODRYAS (MARTENS)

785. Neritina dubia (Chemn.), E. Indies, Phil., New Caledonia. 
SECTION THEODOXUS (MONTF.)

786. Neritina fluviatilis (Linn.), Northern and Middle Europe, Italy, Dalmatia.

787. Neritina Numidica? (Recluz), Algeria. SECTION SMARAGDIA (ISSEL.)

788. Neritina viridis (Linn.), Flor., W. I., Mediterranean. SECTION CLYPEOLUM (RECLUZ.)

789. Neritina pulligera (L.), E. Ind., Austr., Cent. Poly.

79o. Neritina punctulata (Lam.), W. I., Cen. Amer., Panama, Mazatlan, New Granada, Bay of Montija.

791. Neritina Afra (Sowb.), W. Coast of Africa. SECTION NERITONA (MARTENS)

792. Neritina labiosa (Sowb.), Celebes, Phil.

793. Neritina granosa (Sowb.), Sandw. Is. SUB-GENUS CLITHON (MONTF.)

794. Neritina longispina (Recluz.), Maur., Isle of Bourbon, Rodriguez, Madagascar.

795. Neritina spinosa (Budgin), Taheiti.

796. Neritina diadema (Recluz.), E. Ind., Phil.

797. Neritina brevispina (Lam.), E. Ind., Cent. Pac.

SUB-GENUS NERIPTERON (LESSON.) Viti Is.

798. Neritina auriculata (Lam.), Ceylon, E. Ind., Phil.,

799. Neritina Tahitensis (Lesson), Taheiti, Samoa, Sandw. Is.

SECTION ALINA (RECLUZ.)

8oo. Neritina cariosa (Gray), Sandw. Is.

8or. Neritina latissima (Brod.), W. coast of Cen. America.

\section{GENUS NAVICELLA (LAM.)}

Section Cimber (Montf.)

802. Navicella Borbonica (Bory.), Maur., Isle of Bourbon, Madagascar, Seychelles.

8o3. Navicella Cummingiana (Recluz.), Phil.

SECTION ELARA (H. \& A. AD.)

804. Navicella suborbicularis (Sowb.), Andaman Is., Java, and Phil. 
SECTION STENOPOMA (GRAY)

805. Navicella lineata (Lam.), Phil, Viti Is.

FAMILY NERITOPSIDAE

Genus Neritopsis (Grat.)

8o6. Neritopsis radula (Linn.), Sing., Java, Mauritius. FAMILY LIOTIIDAE.

Genus Liotia (Gray), Section Arene (H. \& A. AD.)

807. Liotia radiata (Kiener), W. I.

FAMILY TURBINIDAE.

Sub-Family Phasianellinae, Genus Phasianella (Lam.), SubGenus Phasianella.

SoS. Phasianella australis (Gmelin), S. African and Australian Provinces.

809. Phasianella Aethiopica (Phil.), Seychelles, Australia E. Coast of Africa, Zanzibar.

\section{SUB-GENUS TRICOLIA (RISSO)}

809 I-2 Phasianella tenuis, (Mechand), Med. and Adriatic At. Coast of France.

\section{SUB-FAMILY TURBININAE}

Genus Turbo (Linn.), Sub-Genus Turbo.

8ro. Turbo mannoratus (Linn.) [one polished, also opercula], Ind. O., Phil., Seychelles, Japan.

8I r. Turbo imperialis (Gmel.), Ind. O., China Seas.

812. Turbo petholatus (Linn.), Red S., Ind. O., New Caledonia, Phil.

813. Turbo argyrostomus (Linn.), Seychelles, Ins. Reunion and Annaa, Pac. O. East In.

814. Turbo lajonkairii (Desk.), Keeling Id., Ind. O.,

815. 'Turbo cornutus (Gmel.), Japanese Seas. SUB-GENUS CALLOPOMA (GRAY)

816. Operculum of Turbo fluctuosus (Wood), W. Coast of America, Gulf of California, northward.

\section{SUB-GENUS NINELLA (GRAY)}

81 7. Turbo staminens (Martyn) Var.Lamellosus (Brod.) S. Australia, New Ireland, New Zealand. 
SUB-GENUS MARMOROSTOMA (SWAINS.)

818. Turbo porphrites (Martyn), Ind. O., Phil., New Caledonia, Solomon Is. Seas.

819. Turbo coronatus (Gmel.), Ind O., Jap. and Chinese SUB-GENUS SARMATICUS (GRAY)

820. Turbo sarmaticus (Linn.), Cape region of S. Africa. (GENUS ASTRALIUM (LINK)

Sub-Genus Astralium

821. Astralium longispina (Lam.), W. I., Bahamas, Bermuda, Tortugas, Guaternala.

822. Astralium armatum (Phil.), Antilles. St. Croix. SUB-GENUS LITHOPOMA (GRAY)

822 I-2 Astralium tuber (Linn.), W. I., Florida.

823. Astralium caelatum (Gmel.), W. I., Bahamas. SUB-GENUS GUILDFORDIA (GRAY)

824. Astralium triumphans (Phil.), Japan. SUB-GENUS BOLMA (RISSO)

825. Astralium rugosum (Linn.), Med. S.. At. coast of Spain and S. W. France, Azores, Madeira and Canary.

SUB-GENUS CYCLOCANTHA (SWAINS)

826. Astralium petrosum (Martyn), Var :-confragosum (Gould), Dean's Is., Paumotus group.

827. Astralium senticostatum (Kiener), Ind. O., Austr.?

828. Astralium fimbriatum (Lamb.), Australian Seas, Tasmania.

SUB-GENUS CYCLOCANTHA (SWAINS)

828 I-2 Astralium calcar (Linn.), Ind. O., Austr., Phil., New Caledonia, Java.

SUB-GENUS UVANILLA (GRAY)

829. Astralium olivaceum (Wood), Cen. America, G. of California.

830. Astralium Bushii (Phil.), Panama, G. of Calif.

831. Astralium unguis (Wood), Panama, Mazatlan. SUB-GENUS COOKIA (LESSON)

832. Astralium sulcatum (Martyn), New Zealand, Chatham Is. 


\section{SUB-GENUS POMAULAX (GRAY)}

833. Astralium undosum (Wood), California.

834. Small opercula of Turbos.

FAMILY TROCHIDAE.

Sub-Family Trochinae, Genus Trochus (Linn.), Sub-Genus Trochus.

835. Trochus niloticus (L.), Ind. O., New Ireland, New Caledonia, North Australia.

$$
\text { SUB-GENUS CARDINALIA (GRAY) }
$$

836. Trochus virgatus (Gmelin), Ind. O., Red S. SUB-GENUS TECTUS (MONTF.)

837. Trochus obeliscus (Gmelin.), Ind. and Pac. O., Samoa, Viti, Phil., New Caledonia, N. Austr.

838 . Trochus fenestratus (Gmelin.), Ind. O., Java, Sooloo Is., Phil., Viti and Navigator Is., New Caledonia.

839. Trochus mauritianus (Gmelin.), Ind. O., Madag., Seychelles, Red S., Phil.

SUB-GENUS INFUNDIBULUM (MONTF.)

\section{Section Lamprostoma (Swains.)}

840. Trochus maculatus (Linn.), Phil., Viti Is., Sing., Kingsmill Is., Ind. O.

841. Trochus incrassatus (Lam.), Ceylon, New Caledonia, Sandw. and Viti Is.

842. Trochus sandwichensis (Souleyet), Sandw. Is.

843. Trochus lineatus (Lam.), Austr. Seas.

844. Trochus lineatus (Lam.) Var:- hanleyanus (Reeve) Austr. Seas.

\section{SECTION INFUNDIBULUM.}

845. Trochus chloromphalus (A. Ad.), Jap. Seas, Nagasaki.

SECTION INFUNDIBULOPS (PILSBRY.)

846. Trochus erythraens (Brocchi), Red S.

847. Trochus cariniferus (Beck)[Reeve], Ind. O., Mad., Zanzibar, Red S., Chinese Seas, Liewkiew Is.

SUB-GENUS CLANCULUS (MONTF.)

Section Clanculus.

848. Trochus pharaonius (Linn.), Red S. 
849. Trochus puniceus (Phil.), Zanzibar.

85o. Trochus festivus (Tapparone-Canefri), Bay of Bias, New Guinea.

\section{GENUS MONODONTA (LAM.)}

Sub-Genus Monodonta (Lam.), Section Monodonta.

851. Monodonta labio (Linn.), Var :-confusa (Tapparone Canefri), Singapore.

852. Monodonta australis (Lam.), Seychelles, Madagascar, Cape of G. H. Mascat.

853. Monodonta venniculata (Fischer), Natal, Ins. Socotra SECTION OSILIMUS (PHIL.)

854. Monodonta tamsi (Dunker), Cape Verde and Canary Is., Guinea, C. of G. H.

855. Monodonta punctulata (Lamark), Coasts of Senegal and W. Africa.

GENUS CANTHARIDUS (MONTF.)

Sub-Genus Cantharidus (Montf.), Section Cantharidus (Montf.)

856. Cantharidus iris (Gmel.), New Zealand.

SECTION PHASIANOTROCHUS (FISCHER)

857. Cantharidus nitens (?) (Kiener), Austr. coast, Kangaroo Is.

\section{SUB-GENUS THALOTIA (GRAY)}

Section Thalotia.

858. Cantharidus conicus (Gray), W. and S. Austr., N. Tasmania.

\section{GENUS CHLOROSTOMA (SWAINSON)}

859. Chlorostoma nigricolor (Dunker), Japan.

860. Chlorostoma pellisserpentis (Wood), W. coast of Cen. America, Gulf of California.

86r. Chlorostoma atrum (Lesson), Coasts of Chili and Bolivia.

862. Chlorostoma gruneri (Phil.).

\section{SUB-FAMILY GIBBULINAE}

Genus Gibbula (Risso.), Sub-Genus Gibbula.

863. Gibbula magus (Linn.), Med. and temperate At. coasts of Europe, Azores. 
864. Gibbula obliquata (Gmel.), (?)

GENUS PHOTINULA (H. \& A. AD.) land Is.

865. Photinula taeniata (Wood), Sts. of Magellan, Falk-

\section{GENUS CALLIOSTOMA (SWAINS)}

Sub-Genus Calliostoma.

866. Calliostoma punctulatum (Martyn), Cook's Sts., Dunedin, New Zealand.

867. Calliostoma pellucidum (Valenc.), New Zealand.

868. Calliostoma selectum (Chemn.), New Zealand.

869. Calliostoma flammiger (Dunker), (?)

87o. Calliostoma canaliculatum (Martyn.), Sitka, San Diego, Calif. Lucas.

871. Calliostoma eximium (Reeve), Mazatlan, Cape St.

872. Calliostoma conulum (Linn.), Med. and Adriatic S., Canaries, Madeira, Azores.

SUB-GENUS EUTROCHUS (ARTHUR ADAMS.)

873. Calliostoma jujubinum (Gmel.) Var :-perspectivum (Koch.) [Phil.], Florida, Georgia, E. coast of Honduras.

GENUS ENCHELLUS (PHIL.)

Section Enchellus.

874. Enchellus atratus (Gmelin.), Viti, Nicobar and Fiji Is., Moluc., Sing., Phil., Vanikora, Sorong.

\section{SUB-FAMILY UMBONIUM.}

Genus Umbonium (Link.)

875. Umbonium vestiarium (Linn.), Ind. O., Madras and Ceylon, Java, Phil., Sing., New Ireland.

876. Umbonium callosum (Sowb.), Ind. O. China.

877. Umbonium Thomasi (Crosse), Hakodadi, Jap., N.

878. Umbonium giganteum (Lesson), Japan.

879. Umbonium costatum (Valenc.), Corea, Japan.

880. Umbonium moniliferum (Lam.), Japan.

FAMILY STOMATELLIDAE (A. AD.)

Genus Stomatella (Lam.)

881. Stomatella baconi (A. Ad.), Swan River. 
882. Stomatella sulcifera (Lam.), Phil.

883. Stomatella elegans (Gray), Raine's Is., N. Austr.

GENUS STOMATIA (HELBLING.) [LAM.]

884. Stomatia phymotis (Helbling), Phil., Viti Is., Japan.

885. Stomatia rubra (Lam.), Phil., St. of Corea.

\section{FAMILY HALIOTIDAE}

Genus Haliotis (L.), Section Haliotis.

886. Haliotis Cracherodii (Leach), Fallorones Is., San Diego, Cal.

887. Haliotis corrugata (Gray), San Diego, Cal,, southward, Catalina Is.

888. Haliotis fulgens (Phil.), Lower Cal., Monterey Bay.

889. Haliotis rufescens (Swains), Mendocino Co., San Nicholas Is., Cal. Cal.

89o. Haliotis assimilis (Dall.), Monterey and San Diego,

891. Halioti.: tuberculata (Linn.), Var:- Lamellosa (Lam.), Med. and Adriatic Seas.

892. Haliotis bistriata ( $\mathrm{Gmel}$.), ?

893. Haliotis hauleyana (Sowerby), ?

894. Haliotis speciosa (Reeve), ?

894 I -2 Haliotis roacea (Reeve), W. Coast of Africa.

895. Haliotis varia (Linn.), Austr. and Phil., China, Mozambique, Red S., Maur, Ceylon, Archip., Malay.

896. Haliotis elegans (Koch.), Port Adelaide and W. coast of Austr.

897. Haliotis rugosoplicata (Chemn.), Omaha, Dunedin, New Zealand, Chatham and Huckland Is., S. Austr.

899. Haliotis sanguinea (Hanley), C. of G. H.

899. Haliotis roei (Gray), W. coast of Au tr.

900. Haliotis excavata (Lam.), Austr.

\section{SECTION PADOLLUS (MONTF.)}

901. Haliotis emmae (Gray), Austr.

902. Haliotis tricostalis (Lam.), Austra., Java.

903. Haliotis pulchenima (Martyn.), Paumotus, Lord Hood's Is., S. Austr.

904. Haliotis ovina (Chemn.), Austr., Phil., Navigator Is., Liu-Kiu Is. 
SECTION TEINOTIS (H. \& A. AD.) Austr.

905. Haliotis asinina (Linn.), Jap., China, E. Ind.,

\section{FAMILY FISSURELLIDAE}

Sub-Family Fissurellinae (Pilsbry), Genus Fissurella (Brug.), Sub-Genus Fissurella (Brug.), Section Fissurella.

907. Fissurella costata (Lesson), Valparaiso, Chili.

908. Fissurella crassa (Lam.), Chili.

SECTION CREMIDES (H.\& A. AD.)

W. I.?

909. Fissurella nimbosa (Linn.), Carthagena, Venezuela,

9ro. Fissurella barbardensis (Gmel.), Charlotte Harbor, Florida and Bermuda, Tasmania.

SUB-FAMILY EMARGINULINAE (GILL) [PILSBRY]

Genus Subemarginula (Blainv.), Section Subemarginula.

9ri. Subemarginula imbricata (H. Ad.), Mouth of Victoria River, N. E. Austr.

FAMILY ACMAEIDAE.

Sub-Family Acmaeinae, Genus Acmaea (Eschscholtz)

912. Acmaea testudinalis (Muller), North At. and Pac. and Arctic Oceans.

9r3. Acmaea cubensis (Reeve), St. Thomas, St. Croix, Guadeloupe, etc., W. I., Bahamas. na, Japan.

914. Acmaea saccharina (L.), Viti and Fiji Is.. Amboy-

915. Acmaea lacunosa (Reeve), New Zealand.

GENUS SCURRIA (GRAY)

9r6. Scurria mesolenca (Menke), Cen. Am., G. of Cal. SUB-GENUS LOTTIA (GRAY) [CARPENTER]

917. Scurria gigantea (Gray), San Francisco, Panama. FAMILY PATELLIDAE

Sub-Family Patellinae, Genus Patella (L.), Sub-Genus Patella, Section Patella.

9r8. Patella ferruginea (Gmel.), Med. S., from the Egean, Spain. 

Spain.

919. Patella vulgatá ? (Linn.), Lofoten Is., Norway,

920. Patella conspicua (Phil.), Gaboon, Guinea.

SECTION SCUTELLASTRA (H. \& A. AD.)

921. Patella neglecta (Gray), King George's Sound, Mistaken Is., and Swan River, Austr.

922. Patella barhara (Linn.), ?

923. Patella morbida (Reeve.), C. of G. H.

924. Patella cochlear (Born.), C. of G. H.

925. Patella granatina (Linn.), C. of G. H.

SECTION ANCISTROMESUS (DOLL.)

926. Patella mexicana (Brod. and Sowb.)

SUB-GENUS HELCION (MONTF.)

Section Helcion.

927. Patella pectinata (Born.), C. of G. H.

SECTION PATINA (LEACH) [GRAY]

928. Prtella pellucida (Linn.), Lofoten, Norway, Cascaes Bay, Portugal.

\section{SUB-FAMILY NACELLINAE (THIELE)}

Genus Nacella (Schum.), Section Nacella (Schum.)

929. Nacella mytilina (Helbling) Var:- hyalina (Phil.), Sts. of Magellan, Kerguelen Is.

\section{SECTION PATINELLA (DOLL.)}

930. Nacella aenea (Martyn), Sts. of Magellan. gellan.

931. Nacella aenea Var:-deaurata (Gmel.), Sts. of Ma-

932. Nacella aenea Var:-Magellanica (Gmel.), Sts. of Magellan.

\section{GENUS HELCIONISCUS (DOLL.)} Phil.

933. H lcioniscus articulatus (Reeve), Is. of Ticao,

934. Helcioniscus torenma (Reeve), Nagasaki, Tokio, etc., Jap., China.

935. Helcioniscus radians (Gmel.), New Zealand, Austr.

ORDER POLYPLACOPHORA

936-940. Chiton sp. (?) 


\section{ORDER OPISTHOBRANCHIATA.}

Sub-Order Tectibranchiata, Section Cephalaspidea, Family Actaeonidae (Fischer), Genus Actaeon (Montfort), Section Actaeon (Montfort).

94 I. Actaeon tornatilis (Linn.), Med. and Adriatic Seas, Atlantic from Norway to Morocco.

\section{GENUS SOLIDULA}

94 I I-2 Solidula solidula (L.), Cagayan, Mindanao, Phil.

94I 3-4 Solidula sulcata (Gmel.), Phil., Seychelles, Maur., Red S., N. E. Austr., Sing., Forres St.

\section{GENUS BULLINA (FERUSSAC)}

942. Bullina scabra (Gmelin), Java, Maur., Poly., Austr., N. Zeal., Sandw. I., Japan.

FAMILY SCAPHANDRIDAE (FISCHER)

\section{Genus Scaphander (Montfort)}

943. Scaphander lignarius (Linn.), Med. S., At. O., from Norway to Gibralter.

\section{GENUS ATYS (MONTFORT) \\ Sub-Genus Atys (Montfort)}

944. Atys nancum (Linn.), Sing., Borneo, and Phil., Forres Sts., Viti Is., Red S., Madag.

\section{SECTION ALICULA (EHREUB.)}

945. Atys cylindrica (Helbling), Phil., Forres Sts., Fiji Is., Ceylon, Red. S., Maur., and Seychelles.

FAMILY BULLIDAE (AUCT.) [PILSBRY]

\section{Genus Bulla (Linn.)}

946. Bulla occidentalis (A. AD.), W. I., Flor., Bahamas Trinidad, Vera Cruz.

947. Bulla gouldiana (Pilsbry), San Diego, Cal, Cape St. Lucas, W. Mexico and Mazatlan.

948. Bulla ampulla (Linn.), Viti and Eiji Is., Phil.. Red S.. Seychelles, Madag., Maur.

949. Bulla ampulla Var :-bifasciata (Menke.), Fiji Is., Moluccas, Phil.

950. Bulla australis (Gray) [Quoy and Gaimard] Var :oblonga (A. Ad.), Phil. and Isle of Aunaa, Port Jackson. 
FAMILY AKERIDAE (PILSBRY)

Sub-Family Akerinae (Mazzarelli), Genus Akera (Miller)

951. Akera soluta (Gmel.), Zanzihar, Maur., Ceylon, Phil., New S. Wales, S. Austr.

\section{FAMILY HYDATINIDAE}

Genus Hydatina (Schum)

952. Hydatina physis (Linn.). Natal, Maur., Seychelles, Red S., Phil., Austr., Cuba, W. Africa, Sandw. Is.

953. Hydatina physis (L.) Var :-staminea (Menke), Ind. O., W. I.

954. Hydatina velum (Gmelin), Maur. and Mozambique, Ceylon, and E. I.

\section{.SUB-GENUS AMPLUSTRUM (SCHUM)}

955. Hydatina amplustre (Linn.), N. E. Austr., New Cale., Sandw. Is., Maur., Seychelles.

\section{GENUS MICROMELO (PILSBRY)}

956. Micromelo undata (Brug.), St. Thomas, St. Vincent, St. Martin, Cuba.

\section{SECTION ANASPIDEA.}

Family Aplysudae, Sub-Family Dolabellinae (Pilsbry), Genus Dolabella (Lamark).

957. Dolabella scapula (Martyn), E. Austr., N. E. Austr., Maưr., Seychelles, Natal.

DUPLICATES.

I. Murex saxatilis (35)

2. Voluta vespertilio (233)

3. Conus striatus (435)

4. Voluta Turneri (244)

5. Pterocera millepeda (525)

6. Cypraea talpa (546)

7. Cassis exarata (670)

8. Harpa ventricosa $\left(37^{2}\right)$

9. Harpa conoidalis (373)

Io. Harpa nobilis (376)

I I. Perna

I2. Murex radix $\left(3^{8}\right)$

13. Murex nitidus $(37 \mathrm{I}-2)$ [150] 

THIS BOOK IS DUE ON THE LAST DATE STAMPED BELOW

AN INITIAL FINE OF 25 CENTS WILL BE ASSESSED FOR FAILURE TO RETURN THIS BOOK ON THE DATE DUE. THE PENALTY WILL INCREASE TO 50 CENTS ON THE FOURTH DAY AND TO $\$ 1.00$ ON THE SEVENTH DAY OVERDUE.

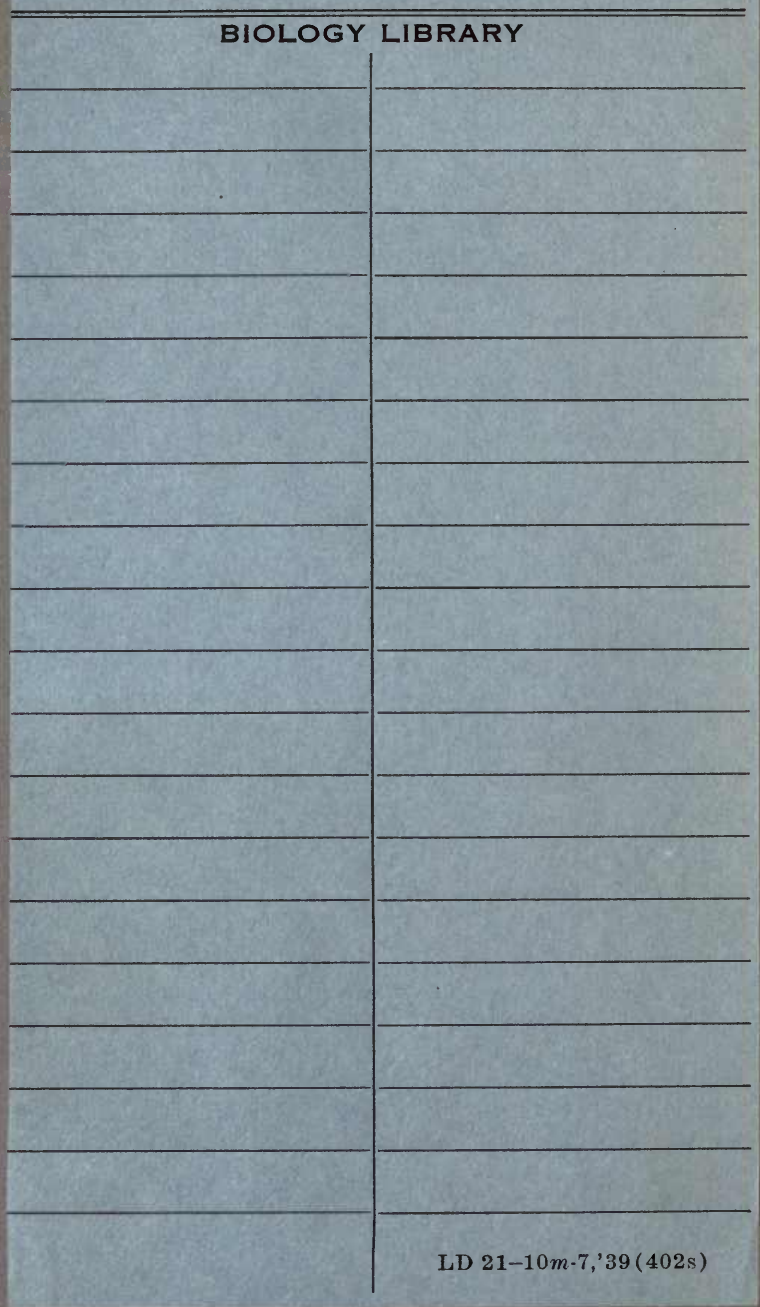


Iyll 10420

Marion natural hist. soc.

QL406 Catalogue of the Doane collection of shells

M35

Biology

APP 261944

$\bar{M} 118498$ m 35

Biology

THE UNIVERSITY OF CALIFORNIA LIBRARY 
